\title{
The existence of positive solution to some asymptotically linear elliptic equations in exterior domains
}

\section{Gongbao Li and Gao-Feng Zheng}

\begin{abstract}
In this paper, we are concerned with the asymptotically linear elliptic problem $-\Delta u+\lambda_{0} u=f(u), u \in H_{0}^{1}(\Omega)$ in an exterior domain $\Omega=\mathbb{R}^{N} \backslash \overline{\mathcal{O}}(N \geqslant 3)$ with $\mathcal{O}$ a smooth bounded and star-shaped open set, and $\lim _{t \rightarrow+\infty} \frac{f(t)}{t}=l, 0<l<+\infty$. Using a precise deformation lemma and algebraic topology argument, we prove under our assumptions that the problem possesses at least one positive solution.
\end{abstract}

\section{Introduction}

In this paper, we are concerned with the existence of positive solution to some asymptotically linear elliptic equations

$$
\left\{\begin{array}{cl}
-\Delta u+\lambda_{0} u=f(u) & \text { in } \Omega \\
u>0 & \text { in } \Omega \\
u=0 & \text { on } \partial \Omega
\end{array}\right.
$$

where $\lambda_{0}>0$ is a constant, $\Omega=\mathbb{R}^{N} \backslash \overline{\mathcal{O}}(N \geqslant 3)$ is an exterior domain with $\mathcal{O}$ a smooth bounded and star-shaped open set.

Problem (1.1) is a special case of the following more general elliptic problem

$$
\left\{\begin{array}{cl}
-\Delta u=f(x, u) & \text { in } \Omega \\
u=0 & \text { on } \partial \Omega,
\end{array}\right.
$$

where $\Omega$ is a bounded or unbounded smooth domain in $\mathbb{R}^{N}(N \geqslant 2)$.

2000 Mathematics Subject Classification: 35J20, 35J25, 35J65.

Keywords: Asymptotically linear elliptic, exterior domain, algebraic topology argument, positive solution. 
Assuming $f(x, 0)=0$, the existence of nontrivial solutions or positive solutions to (1.2) has been studied extensively. Solutions to (1.2) can be viewed as critical points of the functional

$$
I(u)=\frac{1}{2} \int_{\Omega}|\nabla u|^{2} d x-\int_{\Omega} F(x, u) d x
$$

defined on $H_{0}^{1}(\Omega)$, where $F(x, u)=\int_{0}^{u} f(x, s) d s$.

When $\Omega$ is bounded, the Sobolev imbedding

$$
H_{0}^{1}(\Omega) \hookrightarrow L^{q}(\Omega)\left(1 \leqslant q<\frac{2 N}{N-2}\right)
$$

is compact. So if $f(x, u)$ is of "subcritical" at $u=+\infty$, i.e.,

$$
|f(x, u)| \leqslant C\left(1+|u|^{q}\right), \forall(x, u) \in \Omega \times \mathbb{R}^{1}, 2 \leqslant q<2^{*}
$$

and "superlinear" for $u$ at 0 and $+\infty$, i.e.,

$$
\begin{aligned}
\lim _{t \rightarrow 0} \frac{f(x, t)}{t} & =0, \\
\lim _{t \rightarrow+\infty} \frac{f(x, t)}{t} & =+\infty,
\end{aligned}
$$

then $I(u)$ satisfies the so-called Palais-Smale compactness condition (see [1] for the definition) provided that we have the Ambrosetti-Rabinowitz condition: $\exists \theta \in\left(0, \frac{1}{2}\right), u_{0} \geqslant 0$, such that

$$
F(x, u) \leqslant \theta u f(x, u), \forall u \geqslant u_{0}, x \in \Omega .
$$

And then, the critical point of (1.3) can be obtained by Mountain-Pass theorem of Ambrosetti and Rabinowitz (see e.g. [1]). (1.7) guarantees that every (PS) sequence of $I(u)$ is bounded, which is important in verifying the (PS) condition. In the mean time, (1.7) implies (1.6).

A extreme case is the "critical" case, i.e., $q=2^{*}(N>2)$ in (1.4). H. Brezis and L. Nirenberg [6] established a framework to deal with the problem.

Another extreme case is the so-called "asymptotically linear" case, i.e.,

$$
\lim _{t \rightarrow \infty} \frac{f(x, t)}{t}=l, 0<l<+\infty .
$$

For this type of problems, a typical feature is that Ambrosetti-Rabinowitz condition (1.7) does not hold any more. Generally, it is not clear whether any (PS) sequence is bounded or not. For bounded $\Omega,(1.2)$ in asymptotically linear case has been studied by many authors, see e.g. $[3,7,13,16,18,19]$ and the references therein. 
When $\Omega$ is unbounded, say $\Omega=\mathbb{R}^{N}$, problem is more difficult, due to the loss of compactness of Sobolev imbedding caused by the invariance of $\mathbb{R}^{N}$ under the translations and rotations. In the "superlinear" and "subcritical" case, if $f(x, u)=f(u)$ or $f(x, u)=f(|x|, u)$, the existence of a nontrivial solution to (1.1) could be obtained by using radially symmetric Sobolev spaces $H_{r}^{1}\left(\mathbb{R}^{N}\right)$ together with constrained minimization (see e.g. [23], [5]). For general case, the Concentration-Compactness theory by P.L. Lions [21] makes it possible to find at least one nontrivial solution to (1.2) provided some extra conditions about $f(x, u)$ are imposed. A nontrivial solution could be obtained by either "natural constrained minimization" (see [21]) or Mountain-Pass theorem (see [26]). For "asymptotically linear" case when $\Omega=\mathbb{R}^{N}$, we mention that there are a series of works, see [10], [11], [14], [17], [20], [24], [27].

When $\Omega=\mathbb{R}^{N} \backslash \overline{\mathcal{O}}$ where $\mathcal{O}$ is an open bounded domain, problem (1.2) is much more complicated. Because there are no so-called ground state solutions with least energy even if

$$
f(x, u)=|u|^{q-2} u-\lambda u \quad\left(2<q<2^{*}, \lambda>0\right) .
$$

In fact, the infimum

$$
\inf \left\{\int_{\Omega}|\nabla u|^{2}+\lambda u^{2}: \int_{\Omega}|u|^{q} d x=1\right\}
$$

is never achieved if $\Omega=\mathbb{R}^{N} \backslash \overline{\mathcal{O}}(\mathcal{O}$ is a bounded open set $)$. V. Benci and G. Cerami [4] studied the case for

$$
f(x, u)=|u|^{q-2} u-\lambda u\left(2<q<2^{*}, \lambda>0\right),
$$

and obtained the existence of a positive solution provided that $\mathcal{O}$ is of small size. A. Bahri and P.L. Lions studied the case for general exterior domain and got the existence of a positive solution to (1.2) when

$$
f(x, u)=b(x) u^{p}-\lambda_{0} u, \lambda_{0}>0,1<p<\frac{N+2}{N-2}, N>2
$$

by using mainly the topological method. Their works were known for the specialists although they were published much later [2]. Using the similar method as [2], G. Citti generalized the result of [2] to more general $f(x, u)=$ $g(x, u)-\lambda u$, where $g(x, u)$ is "subcritical" and "superlinear" at 0 and $+\infty$ (see [9]).

A natural problem would be as follows: Could one get the existence of a positive solution for problem (1.2) if $\Omega=\mathbb{R}^{N} \backslash \overline{\mathcal{O}}$, and $f(x, u)=g(x, u)-\lambda u$ where $g(x, u)$ is asymptotically linear at $+\infty$ ? 
In this paper we try to answer this question for $g(x, u)=f(u)$, i.e., we want to get the existence of a solution to (1.1). We assume that the function $f(u)$ satisfies the following conditions:

$\left(H_{1}\right) \quad f: \mathbb{R} \rightarrow \mathbb{R}$ is a convex function of $C^{1}$ class and $f(t)=0$ for all $t \leqslant 0 ;$

$\left(H_{2}\right) \quad \lim _{t \rightarrow 0+} \frac{f(t)}{t}=0, \lim _{t \rightarrow+\infty} \frac{f(t)}{t}=l(0<l<+\infty)$;

$\left(H_{3}\right) \quad \frac{f(t)}{t}$ is strictly increasing in $t \in \mathbb{R}^{+}$, and $\frac{f(t)}{t^{p}}$ is decreasing in $t \in \mathbb{R}^{+}$for some $p>1$;

$\left(H_{4}\right) \quad$ For any $M>0$ large enough, there exists $\alpha \in(0, p-1)$ such that $t f^{\prime}(t)-(1+\alpha) f(t) \geqslant 0$ for all $t \in[0,2 M]$.

Our main result is the following

Theorem 1.1 Assume that $\left(H_{1}\right)-\left(H_{4}\right)$ hold with $l>\lambda_{0}$ and the equation at infinity associated to (1.1)

$$
\left\{\begin{array}{c}
-\Delta u+\lambda_{0} u=f(u) \quad \text { in } \mathbb{R}^{N} \\
u \in H^{1}\left(\mathbb{R}^{N}\right)
\end{array}\right.
$$

has a unique positive solution, then (1.1) has at least one solution.

Using the uniqueness theorem for positive solutions to (1.8) by Kwong and Zhang [15] and the method of [9], we have

Corollary 1.2 Assume that $\left(H_{1}\right)-\left(H_{4}\right)$ hold, and let $\theta>0$ be such that $-\lambda_{0} u+f(u)<0$ in $(0, \theta)$ and $-\lambda_{0} u+f(u)>0$ in $(\theta,+\infty)$. If

$$
\frac{-\lambda_{0} u+u f^{\prime}(u)}{-\lambda_{0} u+f(u)} \text { is decreasing in }(\theta,+\infty) \text {, }
$$

then (1.1) has at least one solution.

A typical example of $f(u)$ satisfies $\left(H_{1}\right)-\left(H_{4}\right)$ and the other assumptions in Corollary 1.2 is given by

$$
f(t)=\frac{l t^{2}}{1+t}, t \geqslant 0, l>\lambda_{0}=1
$$

with $p=2$ in $\left(H_{3}\right)$.

To prove our main theorem, we use the argument of contradiction as in [2] and [9]. First of all, we establish a representation theorem (see Proposition 2.1) for the Palais-Smale sequences related to the functional $I$. This 
theorem analyzes the behavior of a (P-S) sequence and states that such a sequence either converges strongly to its weak limit or it differs from it by one or more sequences which after a suitable translation converges to a solution to (1.8). Hence the only obstructions to global compactness in the usual sense are the solutions to (1.8). Because our case is of "asymptotically linear", the standard method, which can easily prove that (P-S) sequences are bounded, can not be applied here. However, we can adapt the method of [17] to prove that any (P-S) sequence is bounded. Then we construct a precise deformation lemma (see Lemma 2.4 ) as in [2,9] under the assumption that (1.1) has no solution and equation (1.8) has a unique positive solution $\omega$. This lemma shows that the energy level-set pair $\left(W_{m}, W_{m-1}\right)$ of the functional $I$ retracts to the pair $\left(W_{m-1}^{\delta}, W_{m-1}\right)$ (see Section 2 for the definition of $W_{m}$ and $\left.W_{m-1}^{\delta}\right)$. Therefore, we can get the relationship between the homologies: $H_{*}\left(W_{m}, W_{m-1}\right) \cong H_{*}\left(W_{m-1}^{\delta}, W_{m-1}\right)$. Furthermore, by the excision property of relative homology group, $H_{*}\left(W_{m}, W_{m-1}\right)=$ $H_{*}\left(W_{m-1}^{\delta} \cap V(m, \varepsilon), W_{m-1} \cap V(m, \varepsilon)\right)$ and the induced homomorphisms

$$
i_{*}: H_{*}\left(W_{m-1}^{\delta} \cap V(m, \varepsilon), W_{m-1} \cap V(m, \varepsilon)\right) \rightarrow H_{*}\left(W_{m}, W_{m-1}\right)
$$

are isomorphisms (for definition of $V(m, \varepsilon)$ see Section 2 below).

Next, by virtue of the properties of the domain $\Omega$, similar to $[2,9]$ we define a map $h$ from $S^{m} \underset{\sigma_{m}}{\times \Delta_{m-1}}$ to $M^{+}$, where $S$ is an $(N-1)$-dimensional sphere in $\Omega, \Delta_{m-1}$ is an $(m-1)$-simplex, $\sigma_{m}$ is a group of permutations of $\{1,2, \ldots, m\}, S^{m} \underset{\sigma_{m}}{\times \Delta_{m-1}}$ is the quotient of $S^{m} \times \Delta_{m-1}$ under the action $\sigma_{m}$, and $M^{+}=\{u \in M: u \geqslant 0\}$ with

$$
M=\left\{u \in H_{0}^{1}(\Omega) \backslash\{0\}: \int_{\Omega}\left(|\nabla u|^{2}+\lambda_{0} u^{2}\right) d x=\int_{\Omega} u f(u) d x\right\},
$$

such that, there exists a $\mu \in \mathbb{N}$ with

(1) $h: S_{0}^{\mu} \underset{\sigma_{\mu}}{\times} \Delta_{\mu-1}^{\eta} \rightarrow W_{\mu-1}$

(2) $h\left(S_{0}^{m} \underset{\sigma_{m}}{\times} \Delta_{m-1}^{\eta}, \partial\left(S_{0}^{m} \underset{\sigma_{m}}{\times} \Delta_{m-1}^{\eta}\right)\right) \subset\left(V\left(m, \varepsilon_{1}\right), W_{m-1}\right) \subset\left(W_{m}, W_{m-1}\right)$,

where $1 \leqslant m \leqslant \mu, S_{0}^{m}$ and $\Delta_{m-1}^{\eta}$ are the appropriate subsets of $S^{m}$ and $\Delta_{m-1}$ respectively. This is a key step for the whole argument. Following [9], it is clear that we need only to define a map $h: S^{m} \times \Delta_{m-1} \rightarrow M^{+}$. For the "superlinear case" as in $[2,9]$, it is trivial to show that $\forall u \in H_{0}^{1}(\Omega)$, there exists a unique $k \in \mathbb{R}^{+}$such that $k u \in M$. Hence

$$
k\left(\varphi \sum_{i=1}^{m} t_{i} \omega\left(\cdot-\lambda x_{i}\right)\right) \varphi \sum_{i=1}^{m} t_{i} \omega\left(\cdot-\lambda x_{i}\right) \in M^{+}
$$


for any $\varphi, m$ and $\lambda$, where $\varphi$ is a cut-off function such that $0 \leqslant \varphi \leqslant 1$, and $\varphi=0$ in $\mathbb{R}^{N} \backslash \Omega, \varphi=1$ in the neighborhood of infinity. But for "asymptotically linear" case, the above statement is not true in general. So we need be more careful. We find that for fixed $\varphi, 1 \leqslant m \leqslant \mu$ and $\lambda>0$ large enough, there does exist a $k\left(\varphi \sum_{i=1}^{m} t_{i} \omega\left(\cdot-\lambda x_{i}\right)\right) \in \mathbb{R}^{+}$such that

$$
k\left(\varphi \sum_{i=1}^{m} t_{i} \omega\left(\cdot-\lambda x_{i}\right)\right) \varphi \sum_{i=1}^{m} t_{i} \omega\left(\cdot-\lambda x_{i}\right) \in M^{+}
$$

(see Lemma 3.2 below). We can then define

$$
h\left(x_{1}, \ldots, x_{m}, t_{1}, \ldots, t_{m}\right)=k\left(\varphi \sum_{i=1}^{m} t_{i} \omega\left(\cdot-\lambda x_{i}\right)\right) \varphi \sum_{i=1}^{m} t_{i} \omega\left(\cdot-\lambda x_{i}\right) \in M^{+},
$$

for any $\left(x_{1}, \ldots, x_{m}, t_{1}, \ldots, t_{m}\right) \in S^{m} \times \Delta_{m-1}$. Hence, using $\left(H_{1}\right)-\left(H_{4}\right)$, we can verify that the map $h$ satisfies the properties (1) and (2) mentioned above.

Finally, using algebraic topological arguments, we obtain that the following diagram is commutative

$$
\begin{array}{ccc}
\mathbb{Z}_{2}=H_{\mu N-1}\left(S_{0}^{\mu} \underset{\sigma_{\mu}}{\times} \Delta_{\mu-1}^{\eta}, \partial\left(S_{0}^{\mu} \underset{\sigma_{\mu}}{\times} \Delta_{\mu-1}^{\eta}\right)\right) & \stackrel{\partial d_{\alpha} \cdots \partial d_{\alpha}}{\longrightarrow} & H_{N-1}(S)=\mathbb{Z}_{2} \\
\downarrow_{\mu N-1} & & \downarrow h_{N-1} \\
H_{\mu N-1}\left(W_{\mu}, W_{\mu-1}\right) & & H_{N-1}\left(W_{1}\right) \\
\downarrow_{\mu N-1}^{-1} & \downarrow i_{N-1}^{-1} \\
H_{\mu N-1}\left(W_{\mu-1}^{\delta} \cap V(\mu, \varepsilon), W_{\mu-1} \cap V(\mu, \varepsilon)\right) & \stackrel{\partial \bar{d}_{\alpha} \cdots \partial \bar{d}_{\alpha}}{\longrightarrow} H_{N-1}\left(W_{0}^{\delta} \cap V(1, \varepsilon)\right)
\end{array}
$$

and that $\partial d_{\alpha}$ is an isomorphism, where $i_{*}^{-1}$ are isomorphisms which we have mentioned above. On one hand, by (1) we know that $h_{\mu N-1}=0$, then $h_{N-1}=0$ by the commutative diagram. On the other hand, there is a function $s: V(1, \varepsilon) \rightarrow S$ such that $s$ is a left inverse of $h$, hence $h_{N-1}$ can not be zero. Thus we show that (1.1) has at least one solution.

Throughout this paper, we always use the standard notations, for instance, $H_{0}^{1}(\Omega)$ is the closure of $C_{0}^{\infty}(\Omega)$ under the norm

$$
\|u\|=\left(\int_{\Omega}\left(|\nabla u|^{2}+\lambda_{0} u^{2}\right) d x\right)^{\frac{1}{2}}
$$

$H^{1}\left(\mathbb{R}^{N}\right)=\left\{u \in L^{2}\left(\mathbb{R}^{N}\right): \nabla u \in L^{2}\left(\mathbb{R}^{N}\right)\right\}$ is a Hilbert space with the norm

$$
\|u\|=\|u\|_{H^{1}\left(\mathbb{R}^{N}\right)}=\left(\int_{\mathbb{R}^{N}}\left(|\nabla u|^{2}+\lambda_{0} u^{2}\right) d x\right)^{\frac{1}{2}} .
$$

We denote $\mathbb{R}^{+} \equiv\{x \in \mathbb{R}: x>0\}$. 


\section{Preliminaries}

It is well known that the solution to (1.1) is a critical point of the functional

$$
I(u)=\frac{1}{2} \int_{\Omega}\left(|\nabla u|^{2}+\lambda_{0} u^{2}\right) d x-\int_{\Omega} F(u) d x
$$

constrained on the Finsler manifold

$$
M=\left\{u \in H_{0}^{1}(\Omega) \backslash\{0\}: \int_{\Omega}\left(|\nabla u|^{2}+\lambda_{0} u^{2}\right) d x=\int_{\Omega} u f(u) d x\right\},
$$

where $F(u)=\int_{0}^{u} f(t) d t$ (see [8] and [17]).

Similarly, the solution to the problem (1.8) at infinity is a critical point of the functional

$$
I_{\infty}(u)=\frac{1}{2} \int_{\mathbb{R}^{N}}\left(|\nabla u|^{2}+\lambda_{0} u^{2}\right) d x-\int_{\mathbb{R}^{N}} F(u) d x
$$

constrained on the Finsler manifold

$$
M_{\infty}=\left\{u \in H^{1}\left(\mathbb{R}^{N}\right) \backslash\{0\}: \int_{\mathbb{R}^{N}}\left(|\nabla u|^{2}+\lambda_{0} u^{2}\right) d x=\int_{\mathbb{R}^{N}} u f(u) d x\right\} .
$$

It is easy to see that

$$
\inf _{M} I(u) \geqslant \inf _{M_{\infty}} I_{\infty}(u) .
$$

By Theorem 1.1 in [17], $\inf _{M_{\infty}} I_{\infty}(u)$ is achieved by some positive $u_{0} \in H^{1}\left(\mathbb{R}^{N}\right)$, which is a ground state solution to (1.8). From now on, we assume (1.8) has a unique positive solution $\omega$. And by the result of [12], we see that $\omega \in C^{\infty}\left(\mathbb{R}^{N}\right)$ is spherically symmetric about some $x_{0} \in \mathbb{R}^{N}$ and if we assume $x_{0}=0$, then $\omega(x)=\omega(r)$ satisfies

$$
\left\{\begin{aligned}
\omega(r) r^{\frac{N-1}{2}} \exp \left(\sqrt{\lambda_{0}} r\right) & \rightarrow c>0 \quad \text { as } r \rightarrow+\infty, \\
\omega^{\prime}(r) r^{\frac{N-1}{2}} \exp \left(\sqrt{\lambda_{0}} r\right) & \rightarrow-c \sqrt{\lambda_{0}} \quad \text { as } r \rightarrow+\infty
\end{aligned}\right.
$$

Since Palais-Smale condition may not be valid in general, we now give a precise compactness result which is similar to [2].

Proposition 2.1 Let $\left\{u_{n}\right\}$ be a nonnegative sequence in $M$ such that $I\left(u_{n}\right) \rightarrow c$ and $\left.d I\right|_{M}\left(u_{n}\right) \rightarrow 0$ as $n \rightarrow \infty$, where $c \geqslant 0$. Then there exist a number $m \in \mathbb{N} \cup\{0\}$ and $m$ sequences $\left\{y_{n}^{j}\right\}_{n \in \mathbb{N}}$ in $\mathbb{R}^{N}(j=1, \ldots, m)$ such that $\left|y_{n}^{j}\right| \rightarrow+\infty$, for every $i \neq j,\left|y_{n}^{i}-y_{n}^{j}\right| \rightarrow+\infty$ as $n \rightarrow \infty$ and

$$
u_{n}(x)=u^{0}(x)+\sum_{i=1}^{m} \omega\left(x-y_{n}^{i}\right)+\psi_{n}(x)
$$

where $u^{0}$ satisfies $d I\left(u^{0}\right)=0, u^{0} \in H_{0}^{1}(\Omega), \psi_{n} \rightarrow 0$ in $H^{1}\left(\mathbb{R}^{N}\right)$ as $n \rightarrow \infty$ and $I\left(u_{n}\right) \rightarrow I\left(u^{0}\right)+m I_{\infty}(\omega)$. 
To prove Proposition 2.1, we need a "Vanishing" lemma. The following "Vanishing" lemma was proved by A. Bahri and P.L. Lions (see Lemma A.1 in Appendix of [2]).

\section{Lemma 2.2 Let}

$$
Q_{k}(t)=\sup _{y \in \mathbb{R}^{N}} \int_{y+B_{t}}\left|\omega_{k}\right|^{2} d x
$$

for all $t \geqslant 0,\left\{\omega_{n}\right\}$ be bounded in $H^{1}\left(\mathbb{R}^{N}\right)$ and assume that for some $t_{0}>0$, $Q_{k}\left(t_{0}\right) \rightarrow 0$. Then $\omega_{k} \rightarrow 0$ in $L^{q}\left(\mathbb{R}^{N}\right)$ for all $2<q<\frac{2 N}{N-2}$. If in addition $\omega_{k}$ satisfies $I^{\prime}\left(\omega_{k}\right) \rightarrow 0$ in $H^{-1}\left(\mathbb{R}^{N}\right)$, then $\omega_{k} \rightarrow 0$ in $H^{1}\left(\mathbb{R}^{N}\right)$ as $k \rightarrow \infty$.

Proof of Proposition 2.1 To be concise, we denote subsequences of a sequence by the sequence itself.

First of all, we should prove that $\left\{u_{n}\right\}$ is bounded in $H_{0}^{1}(\Omega)$. Here we use the argument of [17]. If it were not true, we could assume $\left\|u_{n}\right\| \rightarrow+\infty$ (we always denote

$$
\|u\|=\left(\int_{\Omega}\left(|\nabla u|^{2}+\lambda_{0} u^{2}\right) d x\right)^{\frac{1}{2}}
$$

the equivalent norm of $u \in H_{0}^{1}(\Omega)$, and we denote

$$
\|u\|=\left(\int_{\mathbb{R}^{N}}\left(|\nabla u|^{2}+\lambda_{0} u^{2}\right) d x\right)^{\frac{1}{2}}
$$

for $u \in H^{1}\left(\mathbb{R}^{N}\right)$ if there is no confusion).

There are two cases: (1) $c>0 ;(2) c=0$.

Case (1) $c>0$.

Denote $v_{n}=\frac{2 \sqrt{c} u_{n}}{\left\|u_{n}\right\|}$. So $\left\|v_{n}\right\|=2 \sqrt{c}$. Now we set $\rho_{n}=v_{n}^{2}$. If "Vanishing" occurs, i.e. for any $0<R<+\infty$, as $n \rightarrow \infty$,

$$
\sup _{\mathbb{R}^{N}} \int_{y+B_{R}} \rho_{n}(x) d x \rightarrow 0 .
$$

By virtue of Vanishing Lemma 2.2 (see also [21]),

$$
v_{n} \rightarrow 0 \text { in } L^{\alpha}\left(\mathbb{R}^{N}\right) \text { as } n \rightarrow \infty, 2<\alpha<\frac{2 N}{N-2} .
$$

Assumption $\left(H_{2}\right)$ implies that $\forall \varepsilon>0, \exists C_{\varepsilon}>0$ such that

$$
|f(t)|<\varepsilon|t|+C_{\varepsilon}|t|^{\alpha},|F(t)|<\varepsilon|t|^{2}+C_{\varepsilon}|t|^{\alpha+1}, 1<\alpha<\frac{N+2}{N-2} .
$$


Then (2.3), (2.4) imply that

$$
\int_{\mathbb{R}^{N}} v_{n} f\left(v_{n}\right) d x \rightarrow 0, \int_{\mathbb{R}^{N}} F\left(v_{n}\right) d x \rightarrow 0 \text { as } n \rightarrow \infty .
$$

So

$$
I\left(v_{n}\right)=\frac{1}{2}\left\|v_{n}\right\|^{2}-\int_{\mathbb{R}^{N}} F\left(v_{n}\right) d x=\frac{(2 \sqrt{c})^{2}}{2}+o(1)=2 c+o(1)
$$

On the other hand, for any $t>0$,

$$
\begin{aligned}
I\left(t u_{n}\right) & =\frac{t^{2}}{2}\left\|u_{n}\right\|^{2}-\int_{\mathbb{R}^{N}} F\left(t u_{n}\right) d x, \\
\left\langle I^{\prime}\left(u_{n}\right), u_{n}\right\rangle & =\left\|u_{n}\right\|^{2}-\int_{\mathbb{R}^{N}} u_{n} f\left(u_{n}\right) d x .
\end{aligned}
$$

Then

$$
I\left(t u_{n}\right)=\frac{t^{2}}{2} \int_{\mathbb{R}^{N}} u_{n} f\left(u_{n}\right) d x-\int_{\mathbb{R}^{N}} F\left(t u_{n}\right) d x .
$$

Set $h(t)=\frac{t^{2}}{2} s f(s)-F(t s)$. It is easy to see that

$$
h^{\prime}(t)=\operatorname{tsf}(s)-f(t s) s \begin{cases}\geqslant 0 & \text { if } 0<t \leqslant 1, s>0 \\ \leqslant 0 & \text { if } t \geqslant 1, s>0 \\ =0 & \text { if } t>0, s \leqslant 0\end{cases}
$$

which means that $h(t) \leqslant h(1), \forall t>0$. Then

$$
I\left(t u_{n}\right) \leqslant \frac{1}{2} \int_{\mathbb{R}^{N}} u_{n} f\left(u_{n}\right) d x-\int_{\mathbb{R}^{N}} F\left(u_{n}\right) d x=I\left(u_{n}\right) .
$$

Therefore $I\left(v_{n}\right)=I\left(t_{n} u_{n}\right) \leqslant I\left(u_{n}\right)=c+o(1)$. This is impossible by $(2.5)$. Hence, "Non-vanishing" occurs, i.e. there exists $\eta>0, R>0,\left\{y_{n}\right\} \subset \mathbb{R}^{N}$ such that

$$
\lim _{n \rightarrow \infty} \int_{y_{n}+B_{R}} v_{n}^{2} d x \geqslant \eta>0 .
$$

We distinguish two cases : case (i) $y_{n} \rightarrow y_{0} \in \mathbb{R}^{N}$ as $n \rightarrow \infty$; case (ii) $\left|y_{n}\right| \rightarrow+\infty$ as $n \rightarrow \infty$.

In case (i), we know that there exists $R^{\prime}>R$ such that

$$
\int_{B_{R^{\prime}}} v_{n}^{2} d x \geqslant \frac{\eta}{2}
$$


Since $\left\|v_{n}\right\|=2 \sqrt{c}$, there exists a $v \in H_{0}^{1}(\Omega)$ such that

$$
\left\{\begin{array}{l}
v_{n} \rightarrow v \text { weakly in } H_{0}^{1}(\Omega), \\
v_{n} \rightarrow v \text { strongly in } L_{l o c}^{\alpha}(\Omega), 2 \leqslant \alpha<\frac{2 N}{N-2}, \\
v_{n} \rightarrow v \geqslant 0 \text { a.e. in } \Omega .
\end{array}\right.
$$

(2.7) implies $v \neq 0$. On the other hand, since $\left.d I\right|_{M}\left(u_{n}\right) \rightarrow 0$, we have

$$
\int_{\Omega} \nabla u_{n} \nabla \varphi+\lambda_{0} u_{n} \varphi=\int_{\Omega} f\left(u_{n}\right) \varphi+o(\|\varphi\|) .
$$

Furthermore,

$$
\int_{\Omega} \nabla v_{n} \nabla \varphi+\lambda_{0} v_{n} \varphi=\int_{\Omega} \frac{f\left(2 \sqrt{c} u_{n}\right)}{\left\|u_{n}\right\|} \varphi+o(\|\varphi\|), \forall \varphi \in C_{0}^{\infty}(\Omega) .
$$

Set

$$
p_{n}(x)=\left\{\begin{array}{cl}
\frac{f\left(u_{n}\right)}{u_{n}}, & \text { if } u_{n} \neq 0 \\
0, & \text { if } u_{0}=0
\end{array}\right.
$$

Then

$$
\int_{\Omega} \nabla v_{n} \nabla \varphi+\lambda_{0} v_{n} \varphi=\int_{\Omega} p_{n}(x) v_{n} \varphi, \quad \forall \varphi \in C_{0}^{\infty}(\Omega) .
$$

Since $0 \leqslant p_{n}(x) \leqslant l$, there exists $h \in L_{\text {loc }}^{\infty}(\Omega)$ such that

$$
p_{n}(x) \rightarrow h(x) \text { weakly* in } L_{l o c}^{\infty}(\Omega) .
$$

So $\forall \varphi \in C_{0}^{\infty}(\Omega), \int_{\Omega} p_{n}(x) v_{n}(x) \varphi(x) d x \rightarrow \int_{\Omega} h(x) v \varphi d x$, which combined with (2.8) and (2.9) implies

$$
\int_{\Omega} \nabla v \nabla \varphi+\lambda_{0} v \varphi=\int_{\Omega} h(x) v \varphi, \quad \forall \varphi \in C_{0}^{\infty}(\Omega) .
$$

Denote $A=\{x \in \Omega: v(x)>0\}$. Then $p_{n}(x) \rightarrow l$ a.e. in $A$ as $n \rightarrow \infty$. So $h(x)=l$ in $A$. Therefore, $\forall \varphi \in C_{0}^{\infty}(\Omega)$,

$$
\begin{aligned}
\int_{\Omega} \nabla v \nabla \varphi+\lambda_{0} v \varphi & =\int_{A} h(x) v \varphi+\int_{\Omega \backslash A} h(x) v \varphi \\
& =\int_{A} h(x) v \varphi=l \int_{A} v \varphi=l \int_{\Omega} h(x) v \varphi .
\end{aligned}
$$

Since $\lambda_{0}<l$, we see that $-\Delta$ has an eigenvalue in $H_{0}^{1}(\Omega)$. But the Pohozaev type identity shows that $-\Delta$ has no eigenvalue in $H_{0}^{1}(\Omega)$ since $\Omega=\mathbb{R}^{N} \backslash \overline{\mathcal{O}}$, where $\mathcal{O}$ is a smooth bounded and star-shaped open set (see Theorem 2.12 in Chapter 1 in [22]). 
In case (ii), we set $\widetilde{v}_{n}(x)=v_{n}\left(x+y_{n}\right)$. Then $\left\|\widetilde{v}_{n}\right\|=\left\|v_{n}\right\|$. There exists some $\widetilde{v} \in H_{0}^{1}\left(\mathbb{R}^{N}\right)$ such that

$$
\left\{\begin{array}{l}
\widetilde{v}_{n} \rightarrow \widetilde{v} \text { weakly in } H^{1}\left(\mathbb{R}^{N}\right), \\
\widetilde{v}_{n} \rightarrow \widetilde{v} \text { strongly in } L_{l o c}^{\alpha}\left(\mathbb{R}^{N}\right), 2 \leqslant \alpha<\frac{2 N}{N-2}, \\
\widetilde{v}_{n} \rightarrow \widetilde{v} \geqslant 0 \text { a.e. in } \mathbb{R}^{N} .
\end{array}\right.
$$

(2.6) implies $\widetilde{v} \neq 0$. Since $\left|y_{n}\right| \rightarrow+\infty$, we may assume that $\operatorname{supp} \varphi\left(\cdot-y_{n}\right) \subset \Omega$, $\forall \varphi \in C_{0}^{\infty}\left(\mathbb{R}^{N}\right)$. So by $I^{\prime}\left(u_{n}\right) \rightarrow 0$ in $H^{-1}(\Omega)$ we have

$$
\int_{\Omega}\left(\nabla u_{n} \nabla \varphi\left(x-y_{n}\right)+\lambda_{0} u_{n} \varphi\left(x-y_{n}\right)\right) d x=\int_{\Omega} f\left(u_{n}\right) \varphi\left(x-y_{n}\right) d x+o(1) .
$$

Then $\forall \varphi \in C_{0}^{\infty}\left(\mathbb{R}^{N}\right)$,

$$
\int_{\mathbb{R}^{N}}\left(\nabla \widetilde{u}_{n} \nabla \varphi+\lambda_{0} \widetilde{u}_{n} \varphi\right) d x=\int_{\mathbb{R}^{N}} f\left(\widetilde{u}_{n}\right) \varphi d x+o(1),
$$

where $\widetilde{u}_{n}(x)=u_{n}\left(x+y_{n}\right)$. So

$$
\int_{\mathbb{R}^{N}}\left(\nabla \widetilde{v}_{n} \nabla \varphi+\lambda_{0} \widetilde{v}_{n} \varphi\right) d x=\int_{\mathbb{R}^{N}} \frac{f\left(\widetilde{u}_{n}\right)}{\widetilde{u}_{n}} \widetilde{v}_{n} \varphi d x+o(1) .
$$

Therefore, we can deduce the contradiction similar to case (i). The only difference is that we need Pohozaev type identity on $\mathbb{R}^{N}$ (see [21], [22]) in this case. Hence, $\left\{u_{n}\right\}$ is bounded in $H_{0}^{1}(\Omega)$ if $c>0$.

Case (2) $c=0$.

In this case, $I\left(u_{n}\right) \rightarrow 0, I^{\prime}\left(u_{n}\right) \rightarrow 0$ in $H^{-1}(\Omega)$. For any fixed $\alpha>0$, let $t_{n}=\frac{\alpha}{\left\|u_{n}\right\|}, \omega_{n}=t_{n} u_{n}=\frac{\alpha u_{n}(x)}{\left\|u_{n}\right\|}$. Set $\rho_{n}(x)=\omega_{n}^{2}$. It is easy to rule out "Vanishing" case by a similar argument as in case (1) $c>0$. So "Nonvanishing" occurs, i.e. there exist $\eta>0, R>0$ and $\left\{y_{n}\right\} \subset \mathbb{R}^{N}$ such that

$$
\lim _{n \rightarrow \infty} \int_{y_{n}+B_{R}} \omega_{n}^{2} d x \geqslant \eta>0 .
$$

Set $\widetilde{\omega}_{n}(x)=\omega_{n}\left(x+y_{n}\right)$, then $\left\|\widetilde{\omega}_{n}\right\|=\left\|\omega_{n}\right\|=\alpha$. Then there exists some $\widetilde{\omega} \in H^{1}\left(\mathbb{R}^{N}\right)$ such that

$$
\left\{\begin{array}{l}
\widetilde{\omega}_{n} \rightarrow \widetilde{\omega} \text { weakly in } H^{1}\left(\mathbb{R}^{N}\right), \\
\widetilde{\omega}_{n} \rightarrow \widetilde{\omega} \text { strongly in } L_{l o c}^{2}\left(\mathbb{R}^{N}\right), \\
\widetilde{\omega}_{n} \rightarrow \widetilde{\omega} \geqslant 0 \text { a.e. in } \mathbb{R}^{N} .
\end{array}\right.
$$

By (2.11), we know $\widetilde{\omega} \neq 0$. 
For $n$ large enough, we have

$$
t_{n}=\frac{\alpha}{\left\|u_{n}\right\|} \in(0,1), \quad \frac{f\left(t_{n} u_{n}\right)}{t_{n} u_{n}} \leqslant \frac{f\left(u_{n}\right)}{u_{n}} .
$$

Hence,

$$
\begin{aligned}
\left\|\omega_{n}\right\|^{2}-\int_{\mathbb{R}^{N}} f\left(\omega_{n}\right) \omega_{n} d x & =t_{n}^{2}\left[\left\|u_{n}\right\|^{2}-\int_{\mathbb{R}^{N}} \frac{f\left(t_{n} u_{n}\right)}{t_{n} u_{n}} u_{n}^{2} d x\right] \\
& \geqslant t_{n}^{2}\left[\left\|u_{n}\right\|^{2}-\int_{\mathbb{R}^{N}} \frac{f\left(u_{n}\right)}{u_{n}} u_{n}^{2} d x\right]=0 .
\end{aligned}
$$

Hence, by Fatou's Lemma, we have

$$
\begin{aligned}
I\left(\omega_{n}\right) & =\frac{1}{2}\left\|\omega_{n}\right\|^{2}-\int_{\mathbb{R}^{N}} F\left(\omega_{n}\right)=\int_{\mathbb{R}^{N}}\left[\frac{1}{2} f\left(\omega_{n}\right) \omega_{n}-F\left(\omega_{n}\right)\right] \\
& =\int_{\mathbb{R}^{N}}\left[\frac{1}{2} f\left(\widetilde{\omega}_{n}\right) \widetilde{\omega}_{n}-F\left(\widetilde{\omega}_{n}\right)\right] \geqslant \int_{\mathbb{R}^{N}}\left[\frac{1}{2} f(\widetilde{\omega}) \widetilde{\omega}-F(\widetilde{\omega})\right]+o(1) .
\end{aligned}
$$

But $I\left(\omega_{n}\right)=I\left(t_{n} u_{n}\right) \leqslant I\left(u_{n}\right) \rightarrow 0$. This and (2.12) imply

$$
0 \leqslant \int_{\mathbb{R}^{N}}\left[\frac{1}{2} f(\widetilde{\omega}) \widetilde{\omega}-F(\widetilde{\omega})\right] d x \leqslant 0 .
$$

Then $\widetilde{\omega}=0$. A contradiction.

Now, we have proved that $\left\{u_{n}\right\}$ is bounded in $H_{0}^{1}(\Omega)$. Hence there exists $u \in H_{0}^{1}(\Omega)$ such that

$$
\left\{\begin{array}{l}
u_{n} \rightarrow u \text { weakly in } H_{0}^{1}(\Omega), \\
u_{n} \rightarrow u \text { strongly in } L_{l o c}^{\alpha}(\Omega), 2 \leqslant \alpha<\frac{2 N}{N-2} \\
u_{n} \rightarrow u \text { a.e. in } \Omega
\end{array}\right.
$$

and $f\left(u_{n}\right) \rightarrow f(u)$ a.e. in $\Omega$. Since $|f(t)| \leqslant l|t|$,

$$
\begin{aligned}
\left|\int_{E} f\left(u_{n}\right) \varphi d x\right| & \leqslant\left(\int_{E}\left|f\left(u_{n}\right)\right|^{2} d x\right)^{\frac{1}{2}}\left(\int_{E} \varphi^{2} d x\right)^{\frac{1}{2}} \\
& \leqslant l\left(\int_{E} u_{n}^{2} d x\right)^{\frac{1}{2}}\left(\int_{E} \varphi^{2} d x\right)^{\frac{1}{2}} \leqslant C\left(\int_{E} \varphi^{2} d x\right)^{\frac{1}{2}},
\end{aligned}
$$

for any $\varphi \in C_{0}^{\infty}\left(\mathbb{R}^{N}\right)$ and any measurable set $E \subset \mathbb{R}^{N}$, Vitali's convergence theorem implies

$$
\int_{\mathbb{R}^{N}} f\left(u_{n}\right) \varphi d x \rightarrow \int_{\mathbb{R}^{N}} f(u) \varphi d x, \quad \forall \varphi \in C_{0}^{\infty}\left(\mathbb{R}^{N}\right)
$$


Therefore, by (2.13), (2.14), it is easy to check that $u$ solves $-\Delta u+\lambda_{0} u=$ $f(u)$ in $\Omega$ and $u \geqslant 0$. By the assumptions on $f$, we can easily deduce that for any $\varphi \in C_{0}^{\infty}(\Omega)$,

$$
\left\{\begin{array}{l}
\int_{\mathbb{R}^{N}}\left[F\left(u_{n}\right)-F(u)-F\left(u_{n}-u\right)\right] d x \rightarrow 0 \\
\int_{\mathbb{R}^{N}}\left[f\left(u_{n}\right)-f(u)-f\left(u_{n}-u\right)\right] \varphi d x \rightarrow 0 .
\end{array}\right.
$$

By (2.14), (2.15), we see that we may always assume that $u_{k}$ converges weakly to 0 replacing if necessary $\left\{u_{n}\right\}$ by $\left\{u_{n}-u\right\}$.

Next, in view of Lemma 2.2, either $u_{k} \rightarrow 0$ in $H^{1}\left(\mathbb{R}^{N}\right)$ and the proof is over or there exists an $\alpha>0$ such that we have (up to a subsequence) $Q_{k}\left(t_{0}\right) \geqslant \alpha>0$ and thus there exists $\left\{y_{k}\right\} \subset \mathbb{R}^{N}$ such that

$$
Q_{k}\left(t_{0}\right) \triangleq \int_{y_{k}+B_{t_{0}}}\left|u_{k}\right|^{2} d x \geqslant \alpha>0 .
$$

Therefore, $\widetilde{u}_{k}=u_{k}\left(\cdot+y_{k}\right)$ converges weakly in $H^{1}\left(\mathbb{R}^{N}\right)$ to some $\widetilde{u} \neq 0$ due to Rellich-Kondrakov theorem. Since $u_{k} \rightarrow 0$ in $H^{1}\left(\mathbb{R}^{N}\right)$, we deduce

$$
\left|y_{k}\right| \rightarrow+\infty
$$

But by (2.14)-(2.16), we deduce that $\widetilde{u}$ solves $-\Delta \widetilde{u}+\lambda_{0} \widetilde{u}=f(\widetilde{u})$ in $\mathbb{R}^{N}$, $\widetilde{u} \in H^{1}\left(\mathbb{R}^{N}\right)$ and $-\Delta v_{k}+\lambda_{0} v_{k}=f\left(v_{k}\right)+o(1)$ in $H^{-1}\left(\mathbb{R}^{N}\right)$ where $v_{k}=$ $\widetilde{u}_{k}-\widetilde{u}$. By the uniqueness of the solution to (1.8), we may assume $\widetilde{u}=\omega$. Furthermore, we have

$$
\left\{\begin{array}{l}
\int_{\mathbb{R}^{N}}\left|\nabla u_{k}\right|^{2} d x-\int_{\mathbb{R}^{N}}|\nabla \widetilde{u}|^{2} d x-\int_{\mathbb{R}^{N}}\left|\nabla v_{k}\right|^{2} d x=o(1), \\
\int_{\mathbb{R}^{N}}\left|u_{k}\right|^{2} d x-\int_{\mathbb{R}^{N}}|\widetilde{u}|^{2} d x-\int_{\mathbb{R}^{N}}\left|v_{k}\right|^{2} d x=o(1) .
\end{array}\right.
$$

To conclude, we just iterate the above argument and this iteration procedure has to end in a finite number of steps since we have

$$
m \int_{\mathbb{R}^{N}} \omega^{2} d x \leqslant \liminf _{n \rightarrow \infty} \int_{\mathbb{R}^{N}} u_{k}^{2} d x
$$

Thus $m$ can not go to $\infty$ because of $\omega \neq 0$. We can easily verify that all results of the proposition hold. That completes the proof. 
Corollary 2.3 If (1.1) has no solution, and $\left\{u_{n}\right\}$ satisfies the assumption as in Proposition 2.1, then there exist a number $m \in \mathbb{N} \cup\{0\}$ and $m$ sequences $\left\{y_{n}^{j}\right\}_{n \in \mathbb{N}}$ in $\mathbb{R}^{N}(j=1, \ldots, m)$ such that $\left|y_{n}^{j}\right| \rightarrow+\infty$, for every $i \neq j,\left|y_{n}^{i}-y_{n}^{j}\right| \rightarrow+\infty$ as $n \rightarrow \infty$ and

$$
\begin{cases}u_{n}=\sum_{i=1}^{m} \omega\left(\cdot-y_{n}^{i}\right)+o(1) & \text { as } n \rightarrow \infty \\ I\left(u_{n}\right) \rightarrow m I_{\infty}(\omega) & \text { as } n \rightarrow \infty\end{cases}
$$

Following [2], [9], we define

$$
W_{m}=\left\{u \in M^{+}: I(u) \leqslant(m+1) I_{\infty}(\omega)\right\}
$$

and

$$
V(m, \varepsilon)=\left\{u \in M^{+}:\left\|u-\sum_{i=1}^{m} \omega\left(\cdot-y_{i}\right)\right\|<\varepsilon,\left|y_{i}\right|>\frac{1}{\varepsilon},\left|y_{i}-y_{j}\right|>\frac{1}{\varepsilon}, \forall i \neq j\right\}
$$

where $M^{+}=\{u \in M: u \geqslant 0$ a.e. in $\Omega\}$. From now on, we assume that (1.1) has no solution. So $I(u)>\inf _{M} I(u), \forall u \in M$. This and (2.1) imply $W_{0}=\varnothing$.

For any $u_{0} \in W_{m}$, we consider the Cauchy problem

$$
\left\{\begin{array}{l}
u^{\prime}(t)=-\frac{\left.I\right|_{M} ^{\prime}(u)}{\left(1+\left.|I|_{M}^{\prime}(u)\right|^{2}\right)^{\frac{1}{2}}} \\
u(0)=u_{0} \in W_{m} .
\end{array}\right.
$$

By the standard argument we know that this problem has a unique solution $u\left(t, u_{0}\right)$ for all $t \in \mathbb{R}$ and $u\left(t, u_{0}\right) \in M^{+}$. Denote

$$
T_{\delta}\left(u_{0}\right)=\left\{\begin{array}{l}
\inf \left\{s \geqslant 0: I\left(u\left(s, u_{0}\right)\right) \leqslant m I_{\infty}(\omega)+\delta\right\} \\
+\infty, \quad \text { if the set is empty. }
\end{array}\right.
$$

Then $T_{\delta}\left(u_{0}\right)$ is continuous as a function of $u_{0}$, for every $\delta \geqslant 0$ and if (1.1) has no solution, $T_{\delta}\left(u_{0}\right)<+\infty$ for all $\delta>0$. Next we define $T\left(u_{0}\right)=$ $\min \left\{T_{\delta}\left(u_{0}\right)+\sqrt{\delta}, T_{0}\left(u_{0}\right)\right\}, \quad W_{m-1}^{\delta}=\left\{u\left(T\left(u_{0}\right), u_{0}\right): u_{0} \in W_{m}\right\}$ and $r:$ $[0,1] \times W_{m} \rightarrow W_{m}$ given by $r\left(t, u_{0}\right)=u\left(t T\left(u_{0}\right), u_{0}\right)$. From Corollary 2.3, the following lemma holds (See [2], [9]).

Lemma 2.4 If (1.1) has no solution, then $\left(W_{m}, W_{m-1}\right)$ retracts by deformation through $r$ on $\left(W_{m-1}^{\delta}, W_{m-1}\right)$, and for every $\varepsilon>0, \varepsilon_{1} \in(0, \varepsilon)$ such that $W_{m-1}^{\delta} \backslash W_{m-1} \subset V(m, \varepsilon)$ and $V\left(m, \varepsilon_{1}\right) \subset W_{m-1}^{\delta} \cap V(m, \varepsilon)$. 


\section{Proof of Theorem 1.1}

We will prove the theorem by contradiction as in [2], [9]. Assume that (1.1) has no solution, then the previous arguments are valid.

Let $S$ be an $(N-1)$-dimensional sphere embedded in $\bar{\Omega}$ such that $\lambda S \subset \bar{\Omega}$ for $\lambda \geqslant 1$. Without loss of generality, we assume that $S$ is a sphere of radius 1 . Let $\sigma_{m}$ be the group of permutations of $\{1,2, \ldots, m\}$,

$$
D_{m}=\left\{\left(x_{1}, x_{2}, \ldots, x_{m}\right) \in S^{m}: \exists i \neq j \text { such that } x_{i}=x_{j}\right\},
$$

$\widehat{D}_{m}$ and $\widehat{\widehat{D}}_{m}$ be $\sigma_{m}$-invariant tubular neighborhoods of $D_{m}$, such that $\widehat{D}_{m} \subset \subset$ $\widehat{\widehat{D}}_{m}$ and they retracts by deformation on $D_{m}$. Denote $S_{0}^{m}=\overline{S^{m} \backslash \widehat{D}_{m}}$. We denote

$$
\Delta_{m-1}=\left\{\left(t_{1}, \ldots, t_{m}\right) \in \mathbb{R}^{m}: \sum_{i=1}^{m} t_{i}=1, t_{i} \geqslant 0, \forall i=1, \ldots, m\right\}
$$

and

$$
\Delta_{m-1}^{\eta}=\left\{\left(t_{1}, \ldots, t_{m}\right) \in \Delta_{m-1}: \sup _{1 \leq i \leq m}\left|t_{i}-\frac{1}{m}\right| \leq \eta\right\} .
$$

We will denote the quotient of $S^{m} \times \Delta_{m-1}$ and $S_{0}^{m} \times \Delta_{m-1}$ under the action $\sigma_{m}$ respectively by $S^{m} \underset{\sigma_{m}}{\times \Delta_{m-1}}$ and $S_{0}^{m} \underset{\sigma_{m}}{\times \Delta_{m-1}}$.

Let $\varphi$ be a cut-off function which is a fixed function of class $C^{\infty}$ and identically zero in $\mathbb{R}^{N} \backslash \Omega, 1$ in a neighborhood of $+\infty, 0 \leqslant \varphi \leqslant 1$ in $\mathbb{R}^{N}$.

By the definition of $S_{0}^{m}$ for every $m \in \mathbb{N}$, there exist $\gamma_{m}>0$ and $\tau>0$ such that

$$
\begin{gathered}
\min _{i \neq j}\left|x_{i}-x_{j}\right| \geqslant \gamma_{m}, \quad \forall\left(x_{1}, \ldots, x_{m}\right) \in S_{0}^{m} ; \\
\min _{i \neq j}\left|x_{i}-x_{j}\right| \leqslant \frac{\tau}{2 \sqrt{\lambda_{0}}}, \quad \forall\left(x_{1}, \ldots, x_{m}\right) \in \partial S_{0}^{m} .
\end{gathered}
$$

And we may assume $\tau<\sqrt{\lambda_{0}}$ as long as $\widehat{D}_{m}$ is close enough to $D_{m}$. We claim that there exists $\mu \in \mathbb{N}$ such that

$$
\min _{i \neq j}\left|x_{i}-x_{j}\right| \leqslant \frac{\tau}{2 \sqrt{\lambda_{0}}}, \quad \forall\left(x_{1}, \ldots, x_{\mu}\right) \in S_{0}^{\mu} .
$$

Indeed, if by contradiction, $\forall \mu \in \mathbb{N}, \exists\left(x_{1}, \ldots, x_{\mu}\right) \in S_{0}^{\mu}$ such that $\left|x_{i}-x_{j}\right|>$ $\frac{\tau}{2 \sqrt{\lambda_{0}}}, \forall i \neq j$, then

$$
S \supseteq \bigcup_{i=1}^{\mu}\left(S \cap B\left(x_{i}, \frac{\tau}{4 \sqrt{\lambda_{0}}}\right)\right) .
$$

It is easy to see that the $(N-1)$-measure of $S \cap B\left(x_{i}, \frac{\tau}{4 \sqrt{\lambda_{0}}}\right)$ is independent of $x_{i}$, we may denote it by $C(\tau)$. Then $|S|_{N-1} \geqslant \mu C(\tau)$ which is impossible for $\mu$ large enough. 
The following lemma is important in proving our main result. It can be found in [2], [9].

Lemma 3.1 Let $\varphi \in C\left(\mathbb{R}^{N}\right)$ be radially symmetric and assume that there exist $\alpha, \beta>0, C \in \mathbb{R}$ such that

$$
\begin{aligned}
\varphi(x) \exp (\alpha|x|)|x|^{\beta} & \rightarrow C \quad \text { as } \quad|x| \rightarrow+\infty \\
\int_{\mathbb{R}^{N}}|\psi(x)| \exp (\alpha|x|)\left(1+|x|^{\beta}\right) d x<+\infty &
\end{aligned}
$$

Then

$$
\left(\int_{\mathbb{R}^{N}} \varphi(x+y) \psi(x) d x\right) \exp (\alpha|y|)|y|^{\beta} \rightarrow C \int_{\mathbb{R}^{N}} \psi(x) \exp (-\alpha\langle\gamma, x\rangle) d x
$$

as $|y| \rightarrow+\infty$, for an arbitrary $\gamma \in \mathbb{R}^{N},|\gamma|=1$ (the right hand side is independent of $\gamma$ since $\psi$ is a radial function).

Now, we denote

$$
Q(m, \lambda)=\left\{\varphi \sum_{i=1}^{m} t_{i} \omega\left(\cdot-\lambda x_{i}\right):\left(x_{1}, \ldots, x_{m}, t_{1}, \ldots, t_{m}\right) \in S^{m} \times \Delta_{m-1}\right\}
$$

for $m \leqslant \mu, m \in \mathbb{N}$ and $\lambda>0$;

$$
Q(\lambda)=\bigcup_{m=1}^{\mu} Q(m, \lambda), \text { for } \lambda>0 .
$$

It is obvious that $Q(m, \lambda) \subset Q(\lambda) \subset H_{0}^{1}(\Omega)$.

Lemma 3.2 There exists $\tilde{\lambda}>0$ such that, for all $\lambda \geqslant \tilde{\lambda}$ and $u \in Q(\lambda)$, there is a unique number $k(u) \in \mathbb{R}^{+}$such that $k(u) u \in M$.

Proof. We only need to show that for any $m \in \mathbb{N}$, there is $\lambda_{m}>0$ such that for all $\lambda>\lambda_{m}$ and $u \in Q(m, \lambda)$ there is a unique number $k(u) \in \mathbb{R}^{+}$ such that $k(u) u \in M$.

Define $G: H_{0}^{1}(\Omega) \rightarrow \mathbb{R}$ and $H: S^{m} \times \Delta_{m-1} \rightarrow H_{0}^{1}(\Omega)$ by

$$
G(u)=\int_{\Omega}\left(|\nabla u|^{2}+\lambda_{0} u^{2}\right) d x-l \int_{\Omega} u^{2} d x, \quad \forall u \in H_{0}^{1}(\Omega)
$$

and $\forall\left(x_{1}, \ldots, x_{m}, t_{1}, \ldots, t_{m}\right) \in S^{m} \times \Delta_{m-1}$,

$$
H\left(x_{1}, \ldots, x_{m}, t_{1}, \ldots, t_{m}\right)=\varphi \sum_{i=1}^{m} t_{i} \omega\left(\cdot-\lambda x_{i}\right) .
$$

Clearly, $G$ and $H$ are continuous. 
Now, for any $\left(x_{1}, \ldots, x_{m}, t_{1}, \ldots, t_{m}\right) \in S^{m} \times \Delta_{m-1}$,

$$
\begin{aligned}
G \circ & H\left(x_{1}, \cdots, x_{m}, t_{1}, \ldots, t_{m}\right)= \\
= & \left\|\varphi \sum_{i=1}^{m} t_{i} \omega\left(\cdot-\lambda x_{i}\right)\right\|^{2}-l \int_{\mathbb{R}^{N}}\left(\varphi \sum_{i=1}^{m} t_{i} \omega\left(x-\lambda x_{i}\right)\right)^{2} d x \\
= & \sum_{i, j=1}^{m} t_{i} t_{j} \int_{\mathbb{R}^{N}} \nabla\left(\varphi \omega\left(x-\lambda x_{i}\right)\right) \nabla\left(\varphi \omega\left(x-\lambda x_{j}\right)\right) \\
& +\left(\lambda_{0}-l\right) \varphi^{2} \omega\left(x-\lambda x_{i}\right) \omega\left(x-\lambda x_{j}\right) d x \\
\leqslant & \sum_{i, j=1}^{m} t_{i} t_{j} \int_{\mathbb{R}^{N}} \nabla \omega\left(x-\lambda x_{i}\right) \nabla \omega\left(x-\lambda x_{j}\right)+\lambda_{0} \omega\left(x-\lambda x_{i}\right) \omega\left(x-\lambda x_{j}\right) \\
& -l \varphi^{2} \omega\left(x-\lambda x_{i}\right) \omega\left(x-\lambda x_{j}\right) d x+\int_{\mathbb{R}^{N}}|\Delta \varphi|\left(\sum_{i=1}^{m} t_{i} \omega\left(x-\lambda x_{i}\right)\right)^{2} d x \\
\leqslant & \sum_{i, j=1}^{m} t_{i} t_{j} \int_{\mathbb{R}^{N}} f\left(\omega\left(x-\lambda x_{i}\right)\right) \omega\left(x-\lambda x_{j}\right)-l \omega\left(x-\lambda x_{i}\right) \omega\left(x-\lambda x_{j}\right) d x \\
& +\int_{\mathbb{R}^{N}}\left(|\Delta \varphi|+l\left(1-\varphi^{2}\right)\right)\left(\sum_{i=1}^{m} t_{i} \omega\left(x-\lambda x_{i}\right)\right)^{2} d x .
\end{aligned}
$$

Since $\varphi=1$ in the neighborhood of infinity, we may assume that there exists a ball $B$ such that $\varphi=1$ in $\mathbb{R}^{N} \backslash B$. By (2.2), for $\lambda$ large enough,

$$
\begin{aligned}
\int_{\mathbb{R}^{N}} & \left(|\Delta \varphi|+l\left(1-\varphi^{2}\right)\right)\left(\sum_{i=1}^{m} t_{i} \omega\left(x-\lambda x_{i}\right)\right)^{2} d x \\
& \leqslant C(\varphi, m) \int_{B} \sum_{i=1}^{m} \omega^{2}\left(x-\lambda x_{i}\right) d x \\
& \leqslant C(\varphi, m) \int_{B}\left|x-\lambda x_{i}\right|^{-(N-1)} \exp \left\{-2 \sqrt{\lambda_{0}}\left|x-\lambda x_{i}\right|\right\} d x \\
& \leqslant C(\varphi, m) \int_{B}(\lambda-|x|)^{-(N-1)} \exp \left\{-2 \sqrt{\lambda_{0}}(\lambda-|x|)\right\} d x \\
& =o(1),
\end{aligned}
$$

where $\lim _{\lambda \rightarrow+\infty} O(1)=0$ uniformly with respect to $\left(x_{1}, \ldots, x_{m}, t_{1}, \ldots, t_{m}\right) \in$ $S^{m} \times \Delta_{m-1}$ and $C(\varphi, m)$ is a positive constant which depends only on $\varphi$ and $m$. 
Therefore, for any $\left(x_{1}, \ldots, x_{m}, t_{1}, \ldots, t_{m}\right) \in S^{m} \times \Delta_{m-1}$,

$$
\begin{aligned}
G \circ & H\left(x_{1}, \ldots, x_{m}, t_{1}, \ldots, t_{m}\right) \\
& =\sum_{i, j=1}^{m} t_{i} t_{j} \int_{\mathbb{R}^{N}}\left(f\left(\omega\left(x-\lambda x_{i}\right)\right)-l \omega\left(x-\lambda x_{i}\right)\right) \omega\left(x-\lambda x_{j}\right) d x+o(1) \\
& \leqslant \sum_{i=1}^{m} t_{i}^{2} \int_{\mathbb{R}^{N}}(f(\omega)-l \omega) \omega d x+o(1) \quad\left(\text { by }\left(H_{2}\right),\left(H_{3}\right)\right) .
\end{aligned}
$$

It is easy to see that

$$
\int_{\mathbb{R}^{N}}(f(\omega)-l \omega) \omega d x<0 \quad \text { and } \quad \sum_{i=1}^{m} t_{i}^{2} \geqslant \frac{1}{m} .
$$

So, there exists $\lambda_{m}>0$ such that for any $\left(x_{1}, \ldots, x_{m}, t_{1}, \ldots, t_{m}\right) \in S^{m} \times$ $\Delta_{m-1}$ and $\lambda \geqslant \lambda_{m}$,

$$
G \circ H\left(x_{1}, \ldots, x_{m}, t_{1}, \ldots, t_{m}\right)<0
$$

i.e.,

$$
G(u)<0 \text { for all } u \in Q(m, \lambda) \text { and } \lambda \geqslant \lambda_{m} \text {. }
$$

For any $u \in Q(m, \lambda), \lambda \geqslant \lambda_{m}$, we define

$$
g(t)=\int_{\Omega}\left(|\nabla u|^{2}+\lambda_{0} u^{2}\right) d x-\int_{\Omega} \frac{f(t u)}{t} u d x, \quad t \geqslant 0 .
$$

It is obvious that $g:[0,+\infty) \rightarrow \mathbb{R}$ is continuous and

$$
\begin{aligned}
& g(0)=\lim _{t \rightarrow 0+} g(t)=\int_{\Omega}\left(|\nabla u|^{2}+\lambda_{0} u^{2}\right) d x>0, \\
& \lim _{t \rightarrow+\infty} g(t)=\int_{\Omega}\left(|\nabla u|^{2}+\lambda_{0} u^{2}\right) d x-l \int_{\Omega} u^{2} d x=G(u)<0 \text { by }(3.4) .
\end{aligned}
$$

Therefore there exists a $k \in(0,+\infty)$ such that $g(k)=0$, and it is not difficult to see that such a $k$ is unique by the assumption $\left(H_{3}\right)$ and that $k(u)$ is continuous in $u$. This means that for any $u \in Q(m, \lambda), \lambda \geqslant \lambda_{m}$, there is a unique $k(u) \in \mathbb{R}^{+}$such that $k(u) u \in M$. So the lemma is proved.

From now on, we always assume that $\lambda \geqslant \widetilde{\lambda}, 0 \leqslant m \leqslant \mu$ and denote $\omega_{i}=\omega\left(\cdot-\lambda x_{i}\right)$ if there is no confusion. 
Now, for any $\left(x_{1}, \ldots, x_{m}, t_{1}, \ldots, t_{m}\right) \in S^{m} \underset{\sigma_{m}}{\times} \Delta_{m-1}$, we define a continuous map $h: S^{m} \underset{\sigma_{m}}{\times \Delta_{m-1}} \rightarrow M^{+}$by

$$
h\left(x_{1}, \ldots, x_{m}, t_{1}, \ldots, t_{m}\right)=k\left(\varphi \sum_{i=1}^{m} t_{i} \omega\left(\cdot-\lambda x_{i}\right)\right) \varphi \sum_{i=1}^{m} t_{i} \omega\left(\cdot-\lambda x_{i}\right) .
$$

Denote $B_{m}=h\left(S^{m} \underset{\sigma_{m}}{\times} \Delta_{m-1}\right)$.

It is well known that if $x_{1}, \ldots, x_{m}$ are distinct points in $\mathbb{R}^{N}, \gamma_{1}, \ldots, \gamma_{m} \in \mathbb{R}$ and $\varphi \sum_{j=1}^{m} \gamma_{j} \omega\left(\cdot-x_{j}\right)=0$, then $\gamma_{1}=\gamma_{2}=\cdots=\gamma_{m}=0$ (the proof can be found in [2], [9]). Therefore we have

Lemma 3.3 (i) $B_{m-1} \subset B_{m} \subset M^{+}, \quad \forall m \in \mathbb{N}$;

(ii) $h$ defines an homeomorphism between

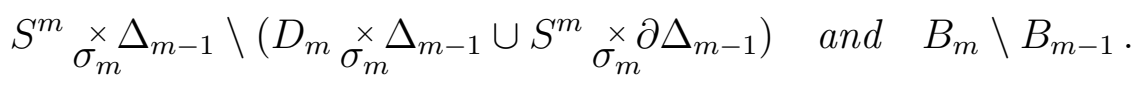

For the sake of not to interrupt proving our main result, the following vital lemma will be proved in the next section.

Lemma 3.4 Let $\varepsilon, \varepsilon_{1}, \delta$ be the real numbers defined in Lemma 2.4. Then there exist $\eta \in[0,1]$ and $\lambda>0$ such that $\forall 1 \leqslant m \leqslant \mu$,

(1) $h: S_{0}^{\mu} \underset{\sigma_{\mu}}{\times} \Delta_{\mu-1}^{\eta} \rightarrow W_{\mu-1}$,

(2) $h\left(S_{0}^{m} \underset{\sigma_{m}}{\times} \Delta_{m-1}^{\eta}, \partial\left(S_{0}^{m} \underset{\sigma_{m}}{\times} \Delta_{m-1}^{\eta}\right)\right) \subset\left(V\left(m, \varepsilon_{1}\right), W_{m-1}\right) \subset\left(W_{m}, W_{m-1}\right)$.

Furthermore $h$ sends any sufficiently small neighborhood of $\partial\left(S_{0}^{m} \underset{\sigma_{m}}{\times} \Delta_{m-1}^{\eta}\right)$ into $W_{m-1}$.

Now we continue our proof of theorem 1.1. Firstly, we mention that all homologies below are with $\mathbb{Z}_{2}$-coefficients. Let $\widehat{\Delta}_{m-1}$ and $\widehat{\widehat{\Delta}}_{m-1}$ with $\widehat{\Delta}_{m-1} \subset \subset \widehat{\widehat{\Delta}}_{m-1}$ be neighborhoods of $\partial \Delta_{m-1}$, which retract by deformation of $\partial \Delta_{m-1}$. Then $\widehat{B}_{m-1} \triangleq h\left(\widehat{D}_{m} \underset{\sigma_{m}}{\times \Delta_{m-1}} \cup S^{m} \underset{\sigma_{m}}{\times \widehat{\Delta}_{m-1}}\right)$ and $\widehat{\widehat{B}}_{m-1} \triangleq$ $h\left(\widehat{\widehat{D}}_{m} \underset{\sigma_{m}}{\times \Delta_{m-1} \cup S^{m}} \underset{\sigma_{m}}{\times \widehat{\widehat{\Delta}}_{m-1}}\right)$ are neighborhoods of $B_{m-1}$ which retract by deformation on $B_{m-1}$. If we denote $t^{\eta}$ the dilatation,

$$
t^{\eta}:\left(\Delta_{m-1}, \partial \Delta_{m-1}\right) \rightarrow\left(\Delta_{m-1}^{\eta}, \partial \Delta_{m-1}^{\eta}\right),
$$

and define $r^{\eta}: S^{m} \times \Delta_{m-1} \rightarrow S^{m} \times \Delta_{m-1}^{\eta}$ by

$$
r^{\eta}(x, y)=\left(x, t^{\eta}(y)\right), \forall(x, y) \in S^{m} \times \Delta_{m-1} .
$$


From Lemma 3.3 and Lemma 3.4, it follows that

$$
h \circ r^{\eta} \circ h^{-1}\left(B_{m} \backslash \widehat{B}_{m-1}, \widehat{\widehat{B}}_{m-1} \backslash \widehat{B}_{m-1}\right) \subset\left(W_{m}, W_{m-1}\right) .
$$

Therefore we can consider the following commutative diagram:

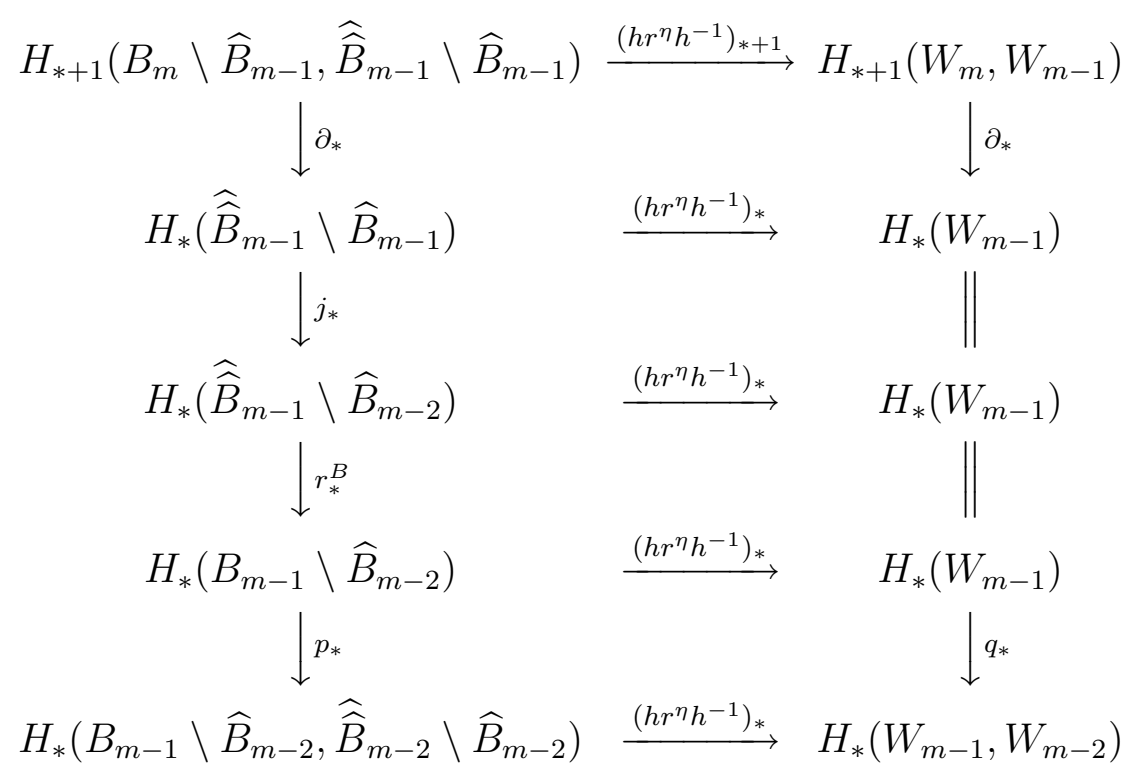

where $\partial_{*}$ are the connecting homomorphisms, $j_{*}$ is induced by the natural embedding, $r_{*}^{B}$ is induced by the retraction of $\widehat{\widehat{B}}_{m-1}$ on $B_{m-1}, p_{*}$ and $q_{*}$ are the projections on the quotients.

Since $h \circ\left(r^{\eta}\right)^{-1}$ is an homeomorphism,

$$
\begin{aligned}
& H_{*}\left(\left(B_{m} \backslash \widehat{B}_{m-1}, \widehat{\widehat{B}}_{m-1} \backslash \widehat{B}_{m-1}\right)\right)
\end{aligned}
$$

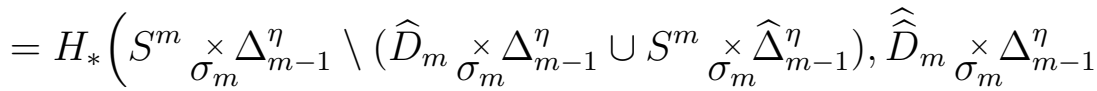

$$
\begin{aligned}
& \left.\cup S^{m} \underset{\sigma_{m}}{\times} \widehat{\widehat{\Delta}}_{m-1}^{\eta} \backslash\left(\widehat{D}_{m} \underset{\sigma_{m}}{\times} \Delta_{m-1}^{\eta} \cup S^{m} \underset{\sigma_{m}}{\times \widehat{\Delta}_{m-1}^{\eta}}\right)\right) \\
& =H_{*}\left(S_{0}^{m} \underset{\sigma_{m}}{\times} \Delta_{m-1}^{\eta}, \partial\left(S_{0}^{m} \underset{\sigma_{m}}{\times} \Delta_{m-1}^{\eta}\right)\right) \text {. }
\end{aligned}
$$

Hence the function $p_{*} \cdot r_{*}^{B} \cdot j_{*} \cdot \partial_{*}$ naturally induces an homomorphism, which we call again $\partial_{*}$,

$$
\begin{aligned}
\partial_{*}: H_{*+1}\left(S_{0}^{m} \underset{\sigma_{m}}{\times} \Delta_{m-1}^{\eta},\right. & \left.\partial\left(S_{0}^{m} \underset{\sigma_{m}}{\times} \Delta_{m-1}^{\eta}\right)\right) \\
& \longrightarrow H_{*}\left(S_{\sigma_{m-1}^{m-1} \times \Delta_{m-2}^{\eta}}^{\eta}, \partial\left(S_{0}^{m-1} \sigma_{m-1}^{\times} \Delta_{m-2}^{\eta}\right)\right) .
\end{aligned}
$$


So the following diagram is commutative

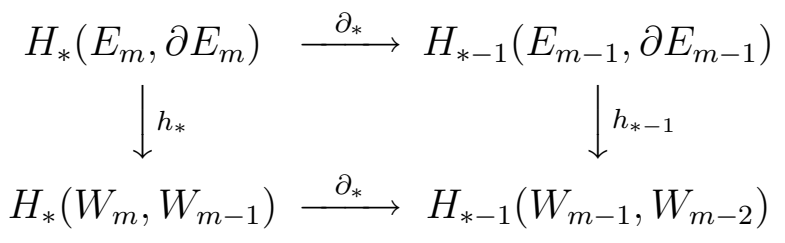

Now, let us denote

$$
E_{m}=S_{0}^{m} \underset{\sigma_{m}}{\times} \Delta_{m-1}^{\eta}, \quad F_{m}=W_{m-1}^{\delta} \cap V(m, \varepsilon), \quad G_{m}=W_{m-1} \cap V(m, \varepsilon) .
$$

From Lemma 2.4 and the excise property of homology groups,

$H_{*}\left(W_{m}, W_{m-1}\right)=H_{*}\left(W_{m-1}^{\delta}, W_{m-1}\right)=H_{*}\left(W_{m-1}^{\delta} \cap V(m, \varepsilon), W_{m-1} \cap V(m, \varepsilon)\right)$.

The natural embedding

$$
i_{*}: H_{*}\left(W_{m-1}^{\delta} \cap V(m, \varepsilon), W_{m-1} \cap V(m, \varepsilon)\right) \rightarrow H_{*}\left(W_{m}, W_{m-1}\right)
$$

is an isomorphism, and it induces an homomorphism

$$
\begin{aligned}
\partial_{*}: & H_{*}\left(W_{m-1}^{\delta} \cap V(m, \varepsilon), W_{m-1} \cap V(m, \varepsilon)\right) \\
& \rightarrow H_{*-1}\left(W_{m-2}^{\delta} \cap V(m-1, \varepsilon), W_{m-2} \cap V(m-1, \varepsilon)\right)
\end{aligned}
$$

such that the following diagram is commutative:

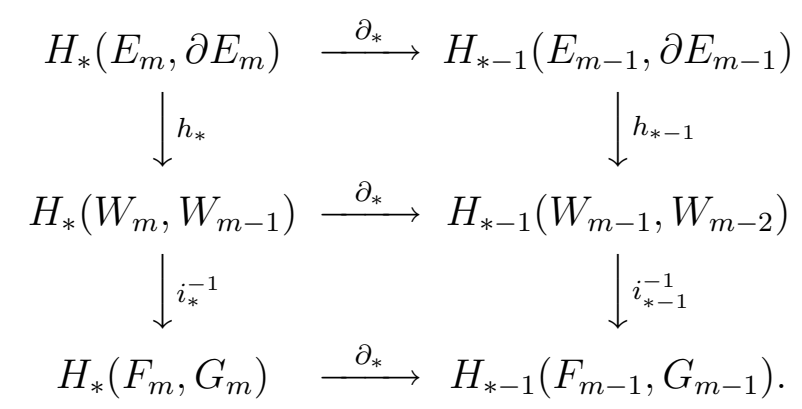

However, by Lemma $3.4(2), h_{*}$ sends $H_{*}\left(S_{0}^{m} \underset{\sigma_{m}}{\times} \Delta_{m-1}^{\eta}, \partial\left(S_{0}^{m} \underset{\sigma_{m}}{\times} \Delta_{m-1}^{\eta}\right)\right)$ to $H_{*}\left(W_{m-1}^{\delta} \cap V(m, \varepsilon), W_{m-1} \cap V(m, \varepsilon)\right)$, so the above diagram simply reduces to

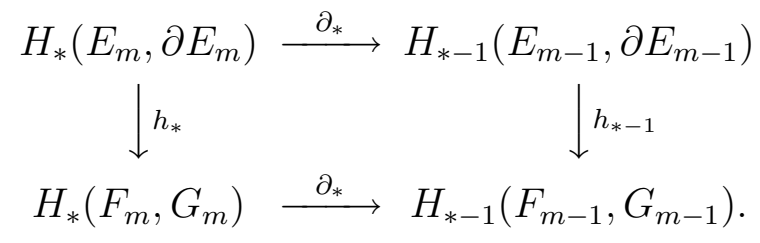


Lemma 3.5 There exists a continuous map

$$
s: V(m, \varepsilon) \longrightarrow S^{m} / \sigma_{m}
$$

such that the following diagram is commutative

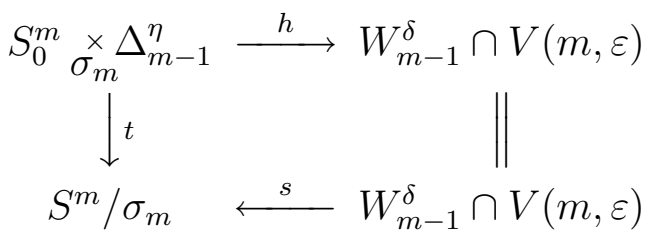

where $t$ is the projection on the first coordinate.

The proof of this lemma is obvious due to Proposition III.1 in [2]. We omit the details.

Let us denote

$$
\text { and } \begin{aligned}
s^{N-1} & : H^{N-1}\left(S^{m} / \sigma_{m}\right) \rightarrow H^{N-1}(V(m, \varepsilon)), \\
t^{N-1}: H^{N-1}\left(S^{m} / \sigma_{m}\right) & \rightarrow H^{N-1}\left(S_{\sigma_{m}}^{\times} \Delta_{m-1}^{\eta}\right)
\end{aligned}
$$

the homomorphisms induced on the cohomology groups. Then for any $\alpha \in$ $H^{N-1}\left(S^{m} / \sigma_{m}\right)$, we can define

$$
\bar{d}_{\alpha}: H_{m N-1}\left(F_{m}, G_{m}\right) \rightarrow H_{m N-N}\left(F_{m}, G_{m}\right)
$$

by $\bar{d}_{\alpha}(\xi)=s^{*}(\alpha) \cap \xi$ and

$$
d_{\alpha}: H_{m N-1}\left(E_{m}, \partial E_{m}\right) \rightarrow H_{m N-N}\left(E_{m}, \partial E_{m}\right)
$$

by $d_{\alpha}(\xi)=t^{*}(\alpha) \cap \xi$, where $\cap$ is the cap product, and we have the following commutative diagram:

$$
\begin{array}{ccc}
H_{m N-1}\left(E_{m}, \partial E_{m}\right) & \stackrel{d_{\alpha}}{\longrightarrow} & H_{m N-N}\left(E_{m}, \partial E_{m}\right) \\
\downarrow h_{m N-1} & & \downarrow h_{m N-N} \\
H_{m N-1}\left(F_{m}, G_{m}\right) & \stackrel{\bar{d}_{\alpha}}{\longrightarrow} & H_{m N-N}\left(F_{m}, G_{m}\right) .
\end{array}
$$

Furthermore, we have $H_{m N-1}\left(S_{0}^{m} \underset{\sigma_{m}}{\times} \Delta_{m-1}^{\eta}, \partial\left(S_{0}^{m} \underset{\sigma_{m}}{\times} \Delta_{m-1}^{\eta}\right)\right)=\mathbb{Z}_{2}$ and there exists $\alpha \in H^{N-1}\left(S^{m} / \sigma_{m}\right)$ such that

$$
\partial \cdot d_{\alpha}: H_{m N-1}\left(E_{m}, \partial E_{m}\right) \rightarrow H_{(m-1) N-1}\left(E_{m-1}, \partial E_{m-1}\right)
$$

sends the generator to the generator (see [2] for details). 
Therefore we have the commutative diagram:

$$
\begin{array}{ccc}
H_{\mu N-1}\left(E_{\mu}, \partial E_{\mu}\right) & \stackrel{\partial d_{\alpha} \cdots \partial d_{\alpha}}{\longrightarrow} & H_{N-1}\left(E_{1}, \partial E_{1}\right) \\
\downarrow h_{\mu N-1} & & \downarrow h_{N-1} \\
H_{\mu N-1}\left(F_{\mu}, G_{\mu}\right) & \stackrel{\partial \bar{d}_{\alpha} \cdots \partial \bar{d}_{\alpha}}{\longrightarrow} & H_{N-1}\left(F_{1}, G_{1}\right) .
\end{array}
$$

Since $W_{0}=\varnothing$ and $S_{0}=S$, the above diagram becomes

$$
\begin{array}{ccc}
H_{\mu N-1}\left(S_{0}^{\mu} \underset{\sigma_{\mu}}{\times} \Delta_{\mu-1}^{\eta}, \partial\left(S_{0}^{\mu} \underset{\sigma_{\mu}}{\times} \Delta_{\mu-1}^{\eta}\right)\right) & \stackrel{\partial d_{\alpha} \cdots \partial d_{\alpha}}{\longrightarrow} & H_{N-1}(S) \\
\downarrow_{\mu N-1} & \downarrow h_{N-1} \\
H_{\mu N-1}\left(W_{\mu-1}^{\delta} \cap V(\mu, \varepsilon), W_{\mu-1} \cap V(\mu, \varepsilon)\right) & \stackrel{\partial \bar{d}_{\alpha} \cdots \partial \bar{d}_{\alpha}}{\longrightarrow} H_{N-1}\left(W_{0}^{\delta} \cap V(1, \varepsilon)\right) .
\end{array}
$$

By Lemma 3.4, $h_{\mu N-1}=0$. By the commutativity of the above diagram and the fact that $\partial d_{\alpha}$ is an isomorphism, we know $h_{N-1}=0$. But this is impossible. Indeed, if $m=1$, then the commutative diagram in Lemma 3.5 implies that $s \circ h=i d$, which implies $s_{*} \circ h_{*}=i d$. Then $h_{N-1}$ is one to one. However, $H_{N-1}(S)=\mathbb{Z}_{2}$. Then $h_{N-1} \neq 0$. A contradiction. We thus complete the proof of Theorem 1.1.

\section{Proof of Lemma 3.4}

First of all, we prove some lemmas.

Lemma 4.1 From the hypotheses we have made on $f$, it follows that

$$
\begin{aligned}
& f(t) \leqslant t f^{\prime}(t) \leqslant p f(t), \forall t \geqslant 0 ; \\
& f\left(\sum_{i=1}^{m} a_{i}\right) \geqslant \sum_{i=1}^{m} f\left(a_{i}\right), \forall a_{i} \geqslant 0, i=1, \ldots, m ; \\
& F\left(\sum_{i=1}^{m} a_{i}\right) \geqslant \sum_{i=1}^{m} F\left(a_{i}\right)+\frac{2^{2+\alpha}-2}{2^{2+\alpha}+\alpha} \sum_{\substack{i, j=1 \\
i \neq j}}^{m} f\left(a_{i}\right) a_{j}, \forall a_{i} \in[0, M]
\end{aligned}
$$

where $M, \alpha$ are given by $\left(H_{4}\right)$.

Proof (4.1) is a direct result of $\left(H_{3}\right)$.

Since $f$ is convex, for every $j \in\{1, \ldots, m\}$, we have

$$
f\left(a_{j}+\sum_{\substack{i=1 \\ i \neq j}}^{m} a_{i}\right) \geqslant f\left(a_{j}\right)+f^{\prime}\left(a_{j}\right) \sum_{\substack{i=1 \\ i \neq j}}^{m} a_{i} .
$$


So

$$
\begin{aligned}
f\left(\sum_{i=1}^{m} a_{i}\right) a_{j} & \geqslant f\left(a_{j}\right) a_{j}+f^{\prime}\left(a_{j}\right) a_{j} \sum_{\substack{i=1 \\
i \neq j}}^{m} a_{i} \\
& \geqslant f\left(a_{j}\right) a_{j}+f\left(a_{j}\right) \sum_{\substack{i=1 \\
i \neq j}}^{m} a_{i}=f\left(a_{j}\right) \sum_{i=1}^{m} a_{i} .
\end{aligned}
$$

Then

$$
f\left(\sum_{i=1}^{m} a_{i}\right) \sum_{j=1}^{m} a_{j} \geqslant \sum_{j=1}^{m} f\left(a_{j}\right) \sum_{i=1}^{m} a_{i} \text {, i.e. } \quad f\left(\sum_{i=1}^{m} a_{i}\right) \geqslant \sum_{j=1}^{m} f\left(a_{j}\right) .
$$

Hence (4.2) is proved.

We only need to show (4.3) when $m=2$, i.e. we will show for any $a, b \in[0, M], F(a+b) \geqslant F(a)+F(b)+\frac{2^{2+\alpha}-2}{2^{2+\alpha}+\alpha}(f(a) b+f(b) a)$.

Indeed, we may assume $a \geqslant b$.

$$
\begin{aligned}
& F(a+b)-F(a)-F(b) \\
& =\int_{0}^{1} f(a+\theta b) b d \theta-\int_{0}^{1} f(\theta b) b d \theta=\int_{0}^{1} \int_{0}^{1} f^{\prime}(t a+\theta b) a b d t d \theta \\
& =\frac{2^{2+\alpha}-2}{2^{2+\alpha}+\alpha} \int_{0}^{1} \int_{0}^{1} f^{\prime}(t a+\theta b) a b d t d \theta+\frac{\alpha+2}{2^{2+\alpha}+\alpha} \int_{0}^{1} \int_{0}^{1} f^{\prime}(t a+\theta b) a b d t d \theta \\
& \geqslant \frac{2^{2+\alpha}-2}{2^{2+\alpha}+\alpha} \int_{0}^{1} \int_{0}^{1} f^{\prime}(t a) a b d t d \theta+\frac{\alpha+2}{2^{2+\alpha}+\alpha} \int_{0}^{1} \int_{0}^{1} f^{\prime}((t+\theta) b) a b d t d \theta \\
& =\frac{2^{2+\alpha}-2}{2^{2+\alpha}+\alpha} f(a) b+\frac{\alpha+2}{2^{2+\alpha}+\alpha} \int_{0}^{1} \int_{t}^{1+t} f^{\prime}(s b) a b d s d t \\
& =\frac{2^{2+\alpha}-2}{2^{2+\alpha}+\alpha} f(a) b+\frac{\alpha+2}{2^{2+\alpha}+\alpha} \int_{0}^{1}(f((1+t) b)-f(t b)) a d t \\
& \geqslant \frac{2^{2+\alpha}-2}{2^{2+\alpha}+\alpha} f(a) b+\frac{\alpha+2}{2^{2+\alpha}+\alpha} \int_{0}^{1}\left((1+t)^{1+\alpha}-t^{1+\alpha}\right) f(b) a d t \quad\left(\text { by } \quad\left(H_{4}\right)\right) \\
& =\frac{2^{2+\alpha}-2}{2^{2+\alpha}+\alpha}(f(a) b+f(b) a) \quad \text { for all } a, b \in[0, M] .
\end{aligned}
$$

Therefore (4.3) is proved.

Lemma $4.2 \forall u \in Q(\lambda)$ and $m>0$, we have

$$
\min \left\{\left(\frac{k(u)}{m}\right)^{p-1}, 1\right\} \leqslant \frac{m^{2} \int_{\Omega}\left(|\nabla u|^{2}+\lambda_{0} u^{2}\right) d x}{\int_{\Omega} f(m u) m u d x} \leqslant \max \left\{\left(\frac{k(u)}{m}\right)^{p-1}, 1\right\} .
$$


Proof. The proof is easy due to Lemma 3.2 and $\left(H_{3}\right)$. We omit the details.

Now, let us proof Lemma 3.4. By (3.1)-(3.3), as in [2], we need only to prove

Lemma 4.3 Suppose that the assumptions in Theorem 1.1 hold, then

$\exists \bar{\lambda}, \bar{\eta}>0$, such that $\forall \lambda \geqslant \bar{\lambda}, \forall \eta \leqslant \bar{\eta}$,

$$
\begin{aligned}
& \text { if } \min \left|x_{i}-x_{j}\right| \geqslant \gamma_{m} \text { and }\left(t_{1}, \ldots, t_{m}\right) \in \Delta_{m-1}^{\eta} \text {, then } \\
& k\left(\varphi \sum_{i=1}^{m} t_{i} \omega\left(\cdot-\lambda x_{i}\right)\right) \varphi \sum_{i=1}^{m} t_{i} \omega\left(\cdot-\lambda x_{i}\right) \in V\left(m, \varepsilon_{1}\right) ;
\end{aligned}
$$

(4.5) $\forall m \geqslant 2, \exists \bar{\lambda}, \bar{\eta}>0$ such that $\forall \lambda \geqslant \bar{\lambda}, \forall \eta \leqslant \bar{\eta}$,

$$
\begin{aligned}
& \text { if } \gamma_{m} \leqslant \min \left|x_{i}-x_{j}\right| \leqslant \frac{\tau}{2 \sqrt{\lambda_{0}}} \text { and }\left(t_{1}, \ldots, t_{m}\right) \in \Delta_{m-1}^{\eta} \text {, then } \\
& I\left(k\left(\varphi \sum_{i=1}^{m} t_{i} \omega\left(\cdot-\lambda x_{i}\right)\right) \varphi \sum_{i=1}^{m} t_{i} \omega\left(\cdot-\lambda x_{i}\right)\right)<m I_{\infty}(\omega) ;
\end{aligned}
$$

(4.6) $\forall m \geqslant 2, \exists \bar{\eta}>0$ such that $\forall \eta \leqslant \bar{\eta}, \exists \bar{\lambda}>0$, such that $\forall \lambda \geqslant \bar{\lambda}$,

$$
\text { if } \gamma_{m} \leqslant \min \left|x_{i}-x_{j}\right| \text { and }\left(t_{1}, \ldots, t_{m}\right) \in \partial \Delta_{m-1}^{\eta} \text {, then }
$$$$
I\left(k\left(\varphi \sum_{i=1}^{m} t_{i} \omega\left(\cdot-\lambda x_{i}\right)\right) \varphi \sum_{i=1}^{m} t_{i} \omega\left(\cdot-\lambda x_{i}\right)\right)<m I_{\infty}(\omega) .
$$

Proof. We will write $k$ instead of $k\left(\varphi \sum_{i=1}^{m} t_{i} \omega_{i}\right)$. Since

$k^{2} \int_{\Omega}\left(\left|\nabla\left(\varphi \sum_{i=1}^{m} t_{i} \omega_{i}\right)\right|^{2}+\lambda_{0}\left(\varphi \sum_{i=1}^{m} t_{i} \omega_{i}\right)^{2}\right) d x=\int_{\Omega} k \varphi \sum_{i=1}^{m} t_{i} \omega_{i} f\left(k \varphi \sum_{i=1}^{m} t_{i} \omega_{i}\right) d x$,

we have

$$
\int_{\Omega}\left(\left|\nabla\left(\varphi \sum_{i=1}^{m} t_{i} \omega_{i}\right)\right|^{2}+\lambda_{0}\left(\varphi \sum_{i=1}^{m} t_{i} \omega_{i}\right)^{2}\right) d x=\int_{\Omega} \frac{\varphi \sum_{i=1}^{m} t_{i} \omega_{i} f\left(k \varphi \sum_{i=1}^{m} t_{i} \omega_{i}\right)}{k} d x
$$

If $\min \left|x_{i}-x_{j}\right| \geqslant \gamma_{m}$, then it is easy to show that

$$
\int_{\Omega}\left(\left|\nabla\left(\varphi \sum_{i=1}^{m} t_{i} \omega_{i}\right)\right|^{2}+\lambda_{0}\left(\varphi \sum_{i=1}^{m} t_{i} \omega_{i}\right)^{2}\right) d x \rightarrow \frac{\|\omega\|^{2}}{m}=\frac{1}{m} \int_{\mathbb{R}^{N}} \omega f(\omega) d x
$$

as $\lambda \rightarrow+\infty$ and $t_{i} \rightarrow \frac{1}{m}$. 
Now, denote

$$
\liminf _{\substack{\lambda \rightarrow+\infty \\ t_{i} \rightarrow 1 / m}} k=k_{1}, \quad \limsup _{\substack{\lambda \rightarrow+\infty \\ t_{i} \rightarrow 1 / m}} k=k_{2} .
$$

Then by (4.7), (4.8), we have

$$
\begin{aligned}
& \frac{1}{m} \int_{\mathbb{R}^{N}} \omega f(\omega) d x=\sum_{i=1}^{m} \int_{\mathbb{R}^{N}} \frac{\frac{1}{m} \omega f\left(k_{1} \frac{1}{m} \omega\right)}{k_{1}} d x=\int_{\mathbb{R}^{N}} \frac{\omega f\left(\frac{k_{1}}{m} \omega\right)}{k_{1}} d x \\
& \frac{1}{m} \int_{\mathbb{R}^{N}} \omega f(\omega) d x=\sum_{i=1}^{m} \int_{\mathbb{R}^{N}} \frac{\frac{1}{m} \omega f\left(k_{2} \frac{1}{m} \omega\right)}{k_{2}} d x=\int_{\mathbb{R}^{N}} \frac{\omega f\left(\frac{k_{2}}{m} \omega\right)}{k_{2}} d x
\end{aligned}
$$

by Lebesgue's Dominated Convergence Theorem and translation. These two equalities become

$$
\int_{\mathbb{R}^{N}} \omega f(\omega) d x=\int_{\mathbb{R}^{N}} \frac{\omega f\left(\frac{k_{1}}{m} \omega\right)}{\frac{k_{1}}{m}} d x=\int_{\mathbb{R}^{N}} \frac{\omega f\left(\frac{k_{2}}{m} \omega\right)}{\frac{k_{2}}{m}} d x .
$$

But by the assumption $\left(H_{3}\right), \frac{k_{1}}{m}=\frac{k_{2}}{m}=1$. Therefore,

$$
\lim _{\substack{\lambda \rightarrow+\infty \\ t_{i} \rightarrow 1 / m}} k=m
$$

and this shows (4.4).

To prove (4.5), we denote $\langle u, v\rangle=\int_{\mathbb{R}^{N}}\left(\nabla u \nabla v+\lambda_{0} u v\right) d x$.

$$
\begin{aligned}
\int_{\Omega}(\mid \nabla & \left.\left.\left(\varphi \sum_{i=1}^{m} k t_{i} \omega_{i}\right)\right|^{2}+\lambda_{0}\left(\varphi \sum_{i=1}^{m} k t_{i} \omega_{i}\right)^{2}\right) d x \\
\leqslant & \left\|\sum_{i=1}^{m} k t_{i} \omega_{i}\right\|^{2}+\int_{\Omega}|\Delta \varphi|\left(\sum_{i=1}^{m} k t_{i} \omega_{i}\right)^{2} d x \\
\leqslant & \sum_{i=1}^{m}\left(k t_{i}\right)^{2}\|\omega\|^{2}+\sum_{\substack{i, j=1 \\
i \neq j}}^{m} t_{i} t_{j} k^{2}\left\langle\omega_{i}, \omega_{j}\right\rangle+C \sum_{i=1}^{m} \int_{\Omega}|\Delta \varphi| \omega_{i}^{2} d x
\end{aligned}
$$

Since $\omega$ is a solution to (1.8), we have

$$
\left\langle\omega_{i}, \omega_{j}\right\rangle=\int_{\mathbb{R}^{N}} f\left(\omega_{i}\right) \omega_{j} d x \text { and }\|\omega\|^{2}=\int_{\mathbb{R}^{N}} f(\omega) \omega d x .
$$

From Lemma 3.1, we have

$$
\sum_{i=1}^{m} \int_{\Omega}|\Delta \varphi| \omega_{i}^{2} d x \leqslant C \exp (-\lambda \tau) \lambda^{-\frac{N-1}{2}} .
$$


Hence, we obtain

$$
\begin{aligned}
& \int_{\Omega}\left(\left|\nabla\left(\varphi \sum_{i=1}^{m} k t_{i} \omega_{i}\right)\right|^{2}+\lambda_{0}\left(\varphi \sum_{i=1}^{m} k t_{i} \omega_{i}\right)^{2}\right) d x \\
& \leqslant \sum_{i=1}^{m}\left(k t_{i}\right)^{2} \int_{\mathbb{R}^{N}} f(\omega) \omega d x+\sum_{\substack{i, j=1 \\
i \neq j}}^{m} t_{i} t_{j} k^{2} \int_{\mathbb{R}^{N}} f\left(\omega_{i}\right) \omega_{j} d x+C \exp (-\lambda \tau) \lambda^{-\frac{N-1}{2}}
\end{aligned}
$$

On the other hand,

$$
\begin{aligned}
\int_{\Omega} F\left(\varphi \sum_{i=1}^{m} k t_{i} \omega_{i}\right) & \geqslant \int_{\mathbb{R}^{N}} F\left(\sum_{i=1}^{m} k t_{i} \omega_{i}\right)-\int_{\mathbb{R}^{N}}\left(F\left(\sum_{i=1}^{m} k t_{i} \omega_{i}\right)-F\left(\varphi \sum_{i=1}^{m} k t_{i} \omega_{i}\right)\right) \\
& \geqslant \int_{\mathbb{R}^{N}} F\left(\sum_{i=1}^{m} k t_{i} \omega_{i}\right)+C \int_{\mathbb{R}^{N}}\left(\varphi^{p}-1\right) F\left(\sum_{i=1}^{m} k t_{i} \omega_{i}\right) \\
& \geqslant \int_{\mathbb{R}^{N}} F\left(\sum_{i=1}^{m} k t_{i} \omega_{i}\right)+C \int_{\mathbb{R}^{N}}\left(\varphi^{p}-1\right) F\left(\omega_{i}\right) \\
& \geqslant \int_{\mathbb{R}^{N}} F\left(\sum_{i=1}^{m} k t_{i} \omega_{i}\right)-C \exp (-\lambda \tau) \lambda^{-\frac{N-1}{2}} .
\end{aligned}
$$

For $\lambda$ large enough and $t_{i}$ close enough to $\frac{1}{m}$, we have $k t_{i} \leqslant 2$ by (4.4). Therefore using the assumption $\left(H_{4}\right)$ and $(4.3)$ for $M=2 \sup _{x \in \mathbb{R}^{N}} \omega(x)$, we have

$$
\begin{aligned}
& \int_{\Omega} F\left(\varphi \sum_{i=1}^{m} k t_{i} \omega_{i}\right) \\
& \geqslant \sum_{i=1}^{m} \int_{\mathbb{R}^{N}} F\left(k t_{i} \omega_{i}\right)+\frac{2^{2+\alpha}-2}{2^{2+\alpha}+\alpha} \sum_{\substack{i, j=1 \\
i \neq j}}^{m} \int_{\mathbb{R}^{N}} f\left(k t_{i} \omega_{i}\right)\left(k t_{j} \omega_{j}\right)-C \exp (-\lambda \tau) \lambda^{-\frac{N-1}{2}} \\
& \geqslant \sum_{i=1}^{m} \int_{\mathbb{R}^{N}} F\left(k t_{i} \omega_{i}\right)+\frac{2^{2+\alpha}-2}{2^{2+\alpha}+\alpha} \sum_{\substack{i, j=1 \\
i \neq j}}^{m} \min \left(1,\left(k t_{i}\right)^{p-1}\right) k t_{j} \int_{\mathbb{R}^{N}} f\left(\omega_{i}\right) \omega_{j} \\
& -C \exp (-\lambda \tau) \lambda^{-\frac{N-1}{2}} .
\end{aligned}
$$

Hence

$$
\begin{aligned}
& I\left(\varphi \sum_{i=1}^{m} k t_{i} \omega_{i}\right) \leqslant \frac{1}{2} \sum_{i=1}^{m}\left(k t_{i}\right)^{2} \int_{\mathbb{R}^{N}} \omega f(\omega)-\sum_{i=1}^{m} \int_{\mathbb{R}^{N}} F\left(k t_{i} \omega\right) \\
& +\sum_{\substack{i, j=1 \\
i \neq j}}^{m}\left(\frac{1}{2} t_{i} k-\frac{2^{2+\alpha}-2}{2^{2+\alpha}+\alpha} \min \left(1,\left(k t_{i}\right)^{p-1}\right)\right) k t_{j} \int_{\mathbb{R}^{N}} f\left(\omega_{i}\right) \omega_{j}+C \exp (-\tau \lambda) \lambda^{-\frac{N-1}{2}} .
\end{aligned}
$$


Since (4.4) holds, for all $i=1, \ldots, m, \quad k t_{i} \rightarrow 1$ as $\lambda \rightarrow+\infty$ and $\eta \rightarrow 0$,

$$
\frac{1}{2} t_{i} k-\frac{2^{2+\alpha}-2}{2^{2+\alpha}+\alpha} \min \left(1,\left(k t_{i}\right)^{p-1}\right) \rightarrow \frac{\alpha+4-2^{2+\alpha}}{2\left(2^{2+\alpha}+\alpha\right)}<0 .
$$

On the other hand, by Lemma 3.1 and $\min \left|x_{i}-x_{j}\right| \leqslant \frac{\tau}{2 \sqrt{\lambda_{0}}}$, it follows that there exist $i, j \in\{1, \ldots, m\}$ such that

$$
\begin{aligned}
\int_{\mathbb{R}^{N}} f\left(\omega_{i}\right) \omega_{j} & \geqslant C \exp \left(-\lambda \sqrt{\lambda_{0}}\left|x_{i}-x_{j}\right|\right)\left(\lambda \sqrt{\lambda_{0}}\left|x_{i}-x_{j}\right|\right)^{-\frac{N-1}{2}} \\
& \geqslant C \exp \left(-\frac{\tau \lambda}{2}\right)\left(\frac{\tau \lambda}{2}\right)^{-\frac{N-1}{2}}
\end{aligned}
$$

Therefore, for $\lambda$ large enough we have

$$
\begin{aligned}
I\left(\varphi \sum_{i=1}^{m} k t_{i} \omega_{i}\right) & <\frac{1}{2} \sum_{i=1}^{m}\left(k t_{i}\right)^{2} \int_{\mathbb{R}^{N}} \omega f(\omega)-\sum_{i=1}^{m} \int_{\mathbb{R}^{N}} F\left(k t_{i} \omega\right) \\
& \leqslant \frac{1}{2} \sum_{i=1}^{m} \int_{\mathbb{R}^{N}} \omega f(\omega)-\sum_{i=1}^{m} \int_{\mathbb{R}^{N}} F(\omega) \\
& =m\left(\frac{1}{2}\|\omega\|^{2}-\int_{\mathbb{R}^{N}} F(\omega)\right)=m I_{\infty}(\omega)
\end{aligned}
$$

i.e. (4.5) is proved.

Finally we prove (4.6). We have

$$
\begin{aligned}
\int_{\Omega} f\left(\varphi \sum_{i=1}^{m} m t_{i} \omega_{i}\right) & \varphi \sum_{i=1}^{m} m t_{i} \omega_{i} \\
= & \int_{\mathbb{R}^{N}} f\left(\sum_{i=1}^{m} m t_{i} \omega_{i}\right) \sum_{i=1}^{m} m t_{i} \omega_{i} \\
& -\int_{\mathbb{R}^{N}}\left(f\left(\sum_{i=1}^{m} m t_{i} \omega_{i}\right)-f\left(\varphi \sum_{i=1}^{m} m t_{i} \omega_{i}\right) \varphi\right) \sum_{i=1}^{m} m t_{i} \omega_{i} \\
\geqslant & \sum_{i=1}^{m} \int_{\mathbb{R}^{N}} f\left(m t_{i} \omega_{i}\right) m t_{i} \omega_{i}+\sum_{\substack{i, j=1 \\
i \neq j}}^{m} \int_{\mathbb{R}^{N}} f\left(m t_{i} \omega_{i}\right) m t_{j} \omega_{j} \\
& +\int_{\mathbb{R}^{N}}\left(\varphi^{p}-1\right) f\left(\sum_{i=1}^{m} m t_{i} \omega_{i}\right) \sum_{i=1}^{m} m t_{i} \omega_{i} \\
\geqslant & \sum_{i=1}^{m} \int_{\mathbb{R}^{N}} f\left(m t_{i} \omega_{i}\right) m t_{i} \omega_{i}-C \exp (-\tau \lambda) \lambda^{-\frac{N-1}{2}}
\end{aligned}
$$


and by Lemma 4.2 and the first part of the proof of (4.5),

$$
\min \left(1,\left(\frac{k}{m}\right)^{p-1}\right) \leqslant \frac{\sum_{i=1}^{m}\left(m t_{i}\right)^{2} \int_{\mathbb{R}^{N}} \omega f(\omega)+o(\lambda)}{\sum_{i=1}^{m} \int_{\mathbb{R}^{N}} m t_{i} \omega f\left(m t_{i} \omega\right)+o(\lambda)}
$$

where $\lim _{\lambda \rightarrow+\infty} \frac{o(\lambda)}{\lambda}=0$. It is not difficult to prove that the function

$$
\left(t_{1}, \ldots, t_{m}\right) \rightarrow \sum_{i=1}^{m}\left(m t_{i}\right)^{2} \int_{\mathbb{R}^{N}} \omega f(\omega)-\sum_{i=1}^{m} \int_{\mathbb{R}^{N}} m t_{i} \omega f\left(m t_{i} \omega\right)
$$

takes a strict maximum on the manifold

$$
\sum_{i=1}^{m} t_{i}=1, t_{i} \geqslant 0, i=1, \ldots, m
$$

at the point $\left(\frac{1}{m}, \ldots, \frac{1}{m}\right)$. Therefore, $\exists \bar{\eta}>0$, such that $\forall \eta<\bar{\eta}, \exists \theta(\eta), \lambda(\eta)$ such that $\forall\left(t_{1}, \ldots, t_{m}\right) \in \partial \Delta_{m-1}^{\eta}, \lambda>\lambda(\eta)$,

$$
\min \left(1,\left(\frac{k}{m}\right)^{p-1}\right) \leqslant \frac{\sum_{i=1}^{m}\left(m t_{i}\right)^{2} \int_{\mathbb{R}^{N}} \omega f(\omega)+o(\lambda)}{\sum_{i=1}^{m} \int_{\mathbb{R}^{N}} m t_{i} \omega f\left(m t_{i} \omega\right)+o(\lambda)}<1-\frac{\theta}{2} .
$$

Consequently, $\forall \eta \leqslant \bar{\eta}$, there exists $\lambda(\eta)$ and $\bar{\theta}(\eta)$ such that $k<m-\bar{\theta}(\eta)$.

But

$$
I\left(\varphi \sum_{i=1}^{m} k t_{i} \omega_{i}\right) \leqslant \frac{1}{2} \sum_{i=1}^{m}\left(k t_{i}\right)^{2} \int_{\mathbb{R}^{N}} \omega f(\omega)-\sum_{i=1}^{m} \int_{\mathbb{R}^{N}} F\left(k t_{i} \omega\right)+o(\lambda) .
$$

The function

$$
\left(t_{1}, \ldots, t_{m}\right) \rightarrow \frac{1}{2} \sum_{i=1}^{m} t_{i}^{2} \int_{\mathbb{R}^{N}} \omega f(\omega)-\sum_{i=1}^{m} \int_{\mathbb{R}^{N}} F\left(t_{i} \omega\right)
$$

takes a strict maximum at the point $(1, \ldots, 1)$. Since

$$
\min \left|x_{i}-x_{j}\right| \geqslant \gamma_{m}
$$

by (4.4), we can assume that $k t_{i}$ belongs to an arbitrary small neighborhood of 1 for all $i=1, \ldots, m$. 
However,

$$
\sum_{i=1}^{m}\left|k t_{i}-1\right| \geqslant m-\sum_{i=1}^{m} k t_{i}=m-k>\bar{\theta}(\eta) .
$$

Hence, $\exists \overline{\bar{\theta}}>0$ such that

$$
\begin{aligned}
I\left(\varphi \sum_{i=1}^{m} k t_{i} \omega_{i}\right) & \leqslant \frac{1}{2} \sum_{i=1}^{m} \int_{\mathbb{R}^{N}} \omega f(\omega)-\sum_{i=1}^{m} \int_{\mathbb{R}^{N}} F(\omega)-\overline{\bar{\theta}}(\eta)+o(\lambda) \\
& <m I_{\infty}(\omega)
\end{aligned}
$$

if $\lambda$ is large enough. Hence (4.6) is proved and we have completed the proof of the lemma.

\section{References}

[1] Ambrosetti, A. And Rabinowitz, P.H.: Dual variational methods in critical point theory and applications. J. Functional Analysis 14 (1973), $349-381$.

[2] Bahri, A. And Lions, P.L.: On the existence of a positive solution of semilinear elliptic equations in unbounded domains. Ann. Inst. H. Poincaré Anal. Non Linéaire 14 (1997), 365-413.

[3] Bartolo, P., Benci, V. and Fortunato, D.: Abstract critical point theorems and applications to some nonlinear problems with "strong" resonance at infinity. Nonlinear Anal. 7 (1983), 981-1012.

[4] Benci, V. and Cerami, G.: Positive solutions of some nonlinear elliptic problems in exterior domains. Arch. Rational Mech. Anal. 99 (1987), no. 4, 283-300.

[5] Berestycki, H. And Lions, P.L.: Nonlinear scalar field equations, I. Existence of a ground state. Arch. Rational. Mech. Anal. 82 (1983), no. 4, 313-345.

[6] Brezis, H. And Nirenberg, L.: Positive solutions of nonlinear elliptic equations involving critical Sobolev exponents. Comm. Pure Appl. Math. 36 (1983), 437-477.

[7] Chang, K.C.: Solutions of asymptotically linear operator equations via Morse theory. Comm. Pure Appl. Math. 34 (1981), 693-712.

[8] Chang, K.C.: Critical point theory and its applications. Shanagi Scientific Technical Press, 1986 (Chinese).

[9] Citsi, G.: On the exterior Dirichlet problem for $\Delta u-u+f(x, u)=0$. Rend. Sem. Mat. Univ. Padova 88 (1992), 83-110. 
[10] Costa, D.G. and Tehrani, H.: On a class of asymptotically linear elliptic problems in $\mathbb{R}^{N}$. J. Differential Equations 173 (2001), 470-494.

[11] Ding, W.Y. And Ni, W.M.: On the existence of positive entire solutions of a semilinear elliptic equation. Arch. Rational Mech. Anal. 91 (1986), $283-308$.

[12] Gidas, B., Ni, W.N. and Nirenberg, L.: Symmetry of positive solutions of nonlinear elliptic equations in $\mathbb{R}^{n}$. In Mathematical analysis and applications, Part A, 369-402. Adv. in Math. Suppl. Stud., 7a. Academic Press, New York-London, 1981.

[13] Hess, P.: Nolinear perturbations of linear elliptic and parabolic problems at resonance: existence of multiple solutions. Ann. Scuola Norm. Sup. Pisa Cl. Sci. (4) 5 (1978), 527-537.

[14] Jeanjean, L.: On the existence of bounded Palais-Smale sequences and application to a Landesmann-Lazer-type problem set on $\mathbb{R}^{N}$. Proc. Roy. Soc. Edinburgh Sect. A 129 (1999), 787-809.

[15] Kwong, M.K. And Zhang, L.: Uniqueness of the positive solution of $\Delta u+f(u)=0$ in annulus. Differential Integral Equations 4 (1991), no. 3, $583-599$.

[16] Li, G. And Zhou, H.-S.: Dirichlet Problem of $p$-Laplacian with Nonlinear Term $f(x, u) \sim u^{p-1}$ at Infinity. In Morse Theory, Minimax Theory and Their Applicatons to Nonlinear Differential Equations, 77-89. Edited by H. Brezis et al. International Press, 2003.

[17] Li, G. And Zhou, H.-S.: The existence of a positive solution to asymptically linear scalar field equations. Proc. Roy. Soc. Edinburgh Sect. A 130 (2000), 81-105.

[18] Li, G. And Zhou, H.-S.: Asymptotically linear Dirichlet problem for the p-Laplacian. Nolinear Anal. 43 (2001), 1043-1055.

[19] Li, G. And Zhou, H.-S.: Multiple solutions to p-Laplacian problems with asymptotic nonlinearity as $u^{p-1}$ at infinity. J. London Math. Soc. (2) 65 (2002) 123-138.

[20] LI, Y.Y.: Nonautonomous nonlinear scalar field equations. Indiana Univ. Math. J. 39 (1990), 283-301.

[21] Lions, P.L.: The concentration-compactness principle in the calculus of variations. The locally compact case. II. Ann. Inst. H. Poincaré Anal. Non Linéaire 1 (1984), 223-283.

[22] Shen, Y.T. And YAn, S.S.: Variational methods in quasilinear elliptic equations. Press of South China Tech. Univ., 1999 (Chinese).

[23] Strauss, W.A.: Existence of solitary waves in higher dimensions. Comm. Math. Phys. 55 (1977), 149-162. 
[24] Stuart, C.A. And Zhou, H.S.: Applying the mountain pass theorem to an asymptotically linear elliptic equation on $\mathbb{R}^{N}$. Comm. Partial Differential Equations 24 (1999), 1731-1758.

[25] YAng, J.F.: Positive solutions of semilinear elliptic problems in exterior domains. J. Differential Equations 106 (1993), 40-69.

[26] Yang, J.F. And Zhu, X.P.: The existence of quasilinear scalar field equation, I. The positive mass cases. Acta Math. Sci. 7 (1987), 341-360.

[27] Zhou, H.S.: Positive solution for a semilinear elliptic equation which is almost linear at infinity. Z. Angew. Math. Phys. 49 (1988), no. 6, 896-906.

Recibido: 21 de abril de 2004

Gongbao Li

Department of Mathematics Huazhong Normal University

Wuhan 430079, P.R. China.

ligb@mail. ccnu. edu.cn

Gao-Feng Zheng

Department of Mathematics Huazhong Normal University Wuhan 430079, P. R. China gfzheng@mail.ccnu.edu.cn

This research is partially supported by NSFC No:10271118 and the National Key Program for Basic Research of China No:2002CCA03700 and the first author is partially supported by Academy of Finland. 